\title{
Campo Volcánico Monogenético Villamaría-Termales, Cordillera Central, Andes colombianos (Parte II): Características composicionales
}

\author{
Pablo Osorio $^{1 *}$; Luis Alvaro Botero-Gómez ${ }^{2,3}$; Hugo Murcia ${ }^{3,4}$; Carlos Borrero ${ }^{3}$; Jeny Alejandra Grajales ${ }^{5}$
}

DOI: http://dx.doi.org/10.18273/revbol.v40n3-2018006 @ (1)

Forma de citar: Osorio, P., Botero-Gómez, L.A., Murcia, H., Borrero, C., y Grajales, J.A. (2018). Campo Volcánico Monogenético Villamaría-Termales, Cordillera Central, Andes colombianos (Parte II): Características composicionales. Boletín de Geología, 40(3), 103-123. DOI: 10.18273/revbol.v40n3-2018006.

\section{RESUMEN}

El Campo Volcánico Monogenético Villamaría-Termales (CVMVT) está compuesto al menos por 14 volcanes monogenéticos, los cuales se encuentran alineados preferencialmente sobre el trazo del Sistema de Fallas Villamaría-Termales (SFVT; flanco occidental de la Cordillera Central de Colombia). Estudios petrográficos y geoquímicos permitieron la caracterización composicional de estos volcanes. Los análisis petrográficos muestran una asociación mineral común para todo el campo, la cual está formada por: piroxeno + anfíbol + plagioclasa; sólo algunos volcanes presentan olivino y otros cuarzo. Los análisis geoquímicos indican una variedad composicional en el CVMVT representada por altos valores en el contenido de $\mathrm{SiO}_{2}$ de $\sim 56$ hasta $\sim 69$ wt.\%, así como una firma adaquítica representada particularmente por valores bajos de Y (10 - 19 ppm) y HREE, y valores altos de Sr (>518 ppm). Al comparar estas características con las del Complejo Volcánico Nevado del Ruiz (CVNR), es posible sugerir que existe una relación genética entre ambos y que posiblemente su origen está asociado a un reservorio común.

Palabras clave: Cordillera Central de Colombia; campo volcánico; vulcanismo monogenético; zonas de subducción; domo de lava; adaquitas.

\section{The Villamaría-Termales Monogenetic Volcanic Field, Central Cordillera, colombian Andes (Part II): Compositional characteristics}

\begin{abstract}
The Villamaría-Termales Monogenetic Volcanic Field (VTMVF) consists of at least 14 monogenetic volcanoes that are aligned along the Villamaria-Termales Fault System (VTFS; western flank of the Central Cordillera of Colombia). Petrography and geochemical data allowed to define the compositional features of this volcanic field. The petrographic analysis shows that: pyroxene + amphibole + plagioclase, is the common mineral association for all the volcanoes from the VTMVF. The presence of other mineral phases such as: olivine and quartz in some volcanoes established the only difference between the whole field. Geochemical data shows a great compositional variety in the VTMVF represented by the $\mathrm{SiO}_{2}$ content between $~ 56$ and $\sim 69$ wt.\%, and an adakitic signature represented by low Y content (10 - $19 \mathrm{ppm})$ and HREE values as well as high $\mathrm{Sr}$ content ( $>518$ ppm). Comparing these characteristics with those from the polygenetic Nevado del Ruiz Volcanic Complex (NRVC), it is possible to suggest a genetic relationship between both and a likely common magmatic reservoir.

Keywords: Colombian Central Cordillera, volcanic field; monogenetic volcanism; subduction zones; lava domes; adakitic-signature.

\footnotetext{
${ }^{1}$ Programa de Geología, Universidad de Caldas, Manizales, Colombia. (*) pablosov@outlook.es

${ }^{2}$ Maestría en Ciencias de la Tierra, Universidad de Caldas, Manizales, Colombia. lualbogo1993@hotmail.com borrero_c@yahoo.com

${ }^{4}$ Departamento de Ciencias Geológicas, Universidad de Caldas, Manizales, Colombia.

${ }^{5}$ Universidad de Concepción, Concepción, Chile. jgrajales@udec.cl
}

${ }^{3}$ Instituto de Investigación en Estratigrafía (IIES), Universidad de Caldas, Manizales, Colombia. hugo.murcia@ucaldas.edu.co;
\end{abstract}




\section{INTRODUCCIÓN}

Los volcanes monogenéticos son un tipo de vulcanismo que se encuentra en todos los ambientes tectónicos: subducción, dorsal oceánica e intraplaca, con preferencia a presentarse en ambientes de intraplaca (Németh, 2010; Murcia, 2015; Cañón-Tapia, 2016). Estos volcanes se clasifican como tal debido a que son producto de una única erupción. Un campo volcánico es un término no-genético utilizado para agrupar múltiples volcanes ubicados en un área específica, teniendo en cuenta factores como su relación genética, relación espaciotemporal y número de centros volcánicos ubicados sobre un área determinada (Cañón-Tapia, 2016).

En los Andes septentrionales (FIGURA 1A), al NW del Complejo Volcánico Nevado del Ruiz (CVNR; $c f$. Martínez et al., 2014), se encuentran una serie de centros volcánicos monogenéticos como domos y flujos de lava, las cuales se encuentran alineados siguiendo el trazo del Sistema de Fallas Villamaría - Termales (SFVT). Este conjunto de volcanes ubicados en los municipios de Manizales y Villamaría (Caldas, Colombia), definen un campo volcánico el cual se ha denominado como Campo Volcánico Monogenético Villamaría - Termales (CVMVT; FIGURA 1B). Así, el CVMVT (FIGURA 1C) hace parte de la Provincia Volcano Tectónica San Diego - Cerro Machín (PVTSC) (cf. Martínez et al., 2014) como una manifestación adicional de vulcanismo monogenético, al igual que los campos volcánicos Samaná al N y Pijaos al S de la Provincia (cf. Borrero et al., 2017; Murcia et al., en prensa) (FIGURA 1B). En este trabajo se presentan las características del CVMVT como un campo volcánico independiente, que aunque puede tener relación con el CVNR (cf. Thouret et al., 1990; Schaefer, 1995; Borrero et al., 2009), registra una historia geológica propia. El CVMVT está compuesto de los siguientes centros eruptivos: domos Sancancio, Tesorito, Gallinazo, Amazonas, Victoria, Sabinas, La Oliva, La Negra, La Esperanza, La Laguna, Santana, El Plato y San Luis, y Flujo de lava de Lusitania (Botero et al., 2018).

En este estudio se presentan, analizan e interpretan los resultados de diversos estudios petrográficos y geoquímicos, con el objetivo de caracterizar composicionalmente los centros volcánicos del CVMVT, creando una base para continuar con el entendimiento de la evolución eruptiva de este campo de manera independiente a la del CVNR.

\section{MARCO GEOLÓGICO}

ElCVMVT se encuentra ubicado sobre distintas unidades litodémicas y litoestratigráficas (FIGURA 2), además de las diferentes estructuras geológicas presentes en el flanco occidental de la Cordillera Central de Colombia, que corresponden principalmente a productos de la compleja actividad tectónica que ha ocurrido en el costado NW de los Andes desde el Jurásico Medio hasta el Presente (Maloney et al., 2013). En esta parte de la Cordillera existen diversas litologías, las cuales resumen también la historia de la formación de Los Andes septentrionales (e.g. Irving, 1971; Restrepo y Toussaint, 1988; Toussaint y Restrepo, 1987). Los volcanes que componen el CVMVT se encuentran principalmente sobre un basamento metamórfico y metasedimentario afectado por intrusiones y fallas regionales como la falla San Jerónimo (Bohórquez et al., 2005), y locales como las fallas Gallinazo y Mangabonita (González y Jaramillo, 2002).

\section{Unidades metamórficas, metasedimentarias y volcanoclásticas Mesozoicas-Paleozoicas}

El Complejo Cajamarca es una unidad litoestratigráfica compuesta por rocas metamórficas de bajo grado de las facies esquisto verde a anfibolita (Maya y González, 1995), con edades de metamorfismo del Triásico ( 240 - $220 \mathrm{Ma})$ según Villagómez et al. (2011) o del Jurásico Superior ( 157-146 Ma) según BlancoQuintero et al. (2014). Esta unidad se encuentra limitada tectónicamente al W por la falla San Jerónimo (McCourt, 1984), que la separa del Complejo Quebradagrande (Maya y González, 1995; Nivia et al., 2006) (FIGURA 2).

El Complejo Quebradagrande (Maya y González, 1995) corresponde a las rocas sedimentarias y volcano-sedimentarias del Cretácico (Villagómez y Spikings, 2013) ubicadas entre los departamentos de Caldas y Antioquia (Colombia). En este Complejo se han identificado dos miembros denominados: Sector Oriental, con la presencia de fósiles de edad Albiana, compuesto por cuerpos métricos de conglomerados con guijos de cuarcitas y fragmentos de rocas metamórficas; y Sector Occidental, compuesto por niveles conglomeráticos con presencia de guijos de composición volcánica (Gómez-Cruz et al., 1995); en este sector es posible también observar un metamorfismo de muy bajo grado (FIGURA 2). 

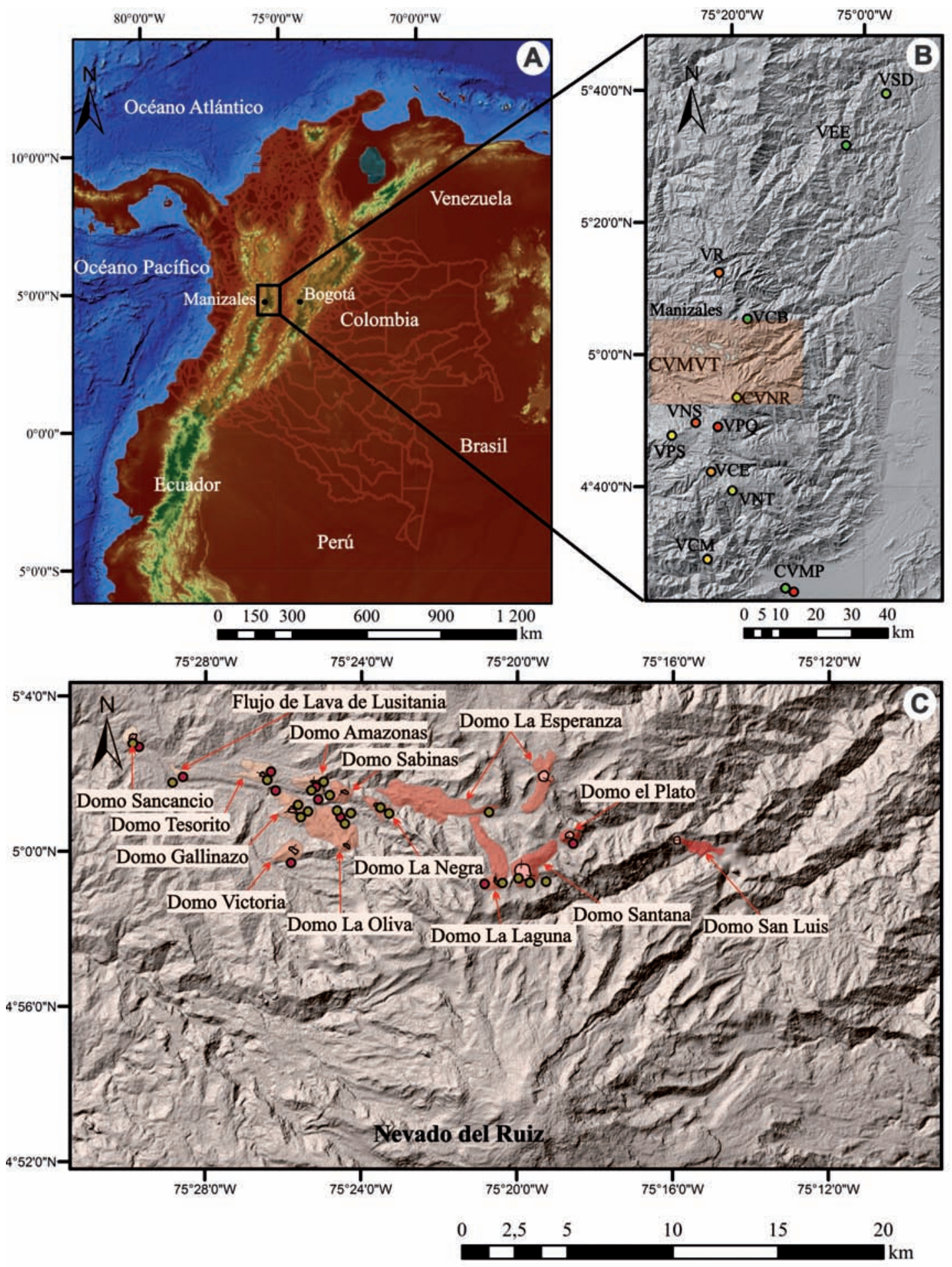

FIGURA 1. A. Localización de la Provincia Volcano Tectónica San Diego - Cerro Machín (PVTSC) en la Cordillera Central de Colombia (rectángulo negro). B. Ubicación de la ciudad de Manizales, el Campo Volcánico Monogenético Villamaría-Termales (CVMVT) y el Complejo Volcánico Nevado del Ruiz (CVNR) dentro de la PVTSC. CVMP: Campo Volcánico Monogenético Pijao, VCM: Volcán Cerro Machín, VNT: Volcán Nevado del Tolima, VCE: Volcán Cerro España, VPS: Volcán Paramillo de Santa Rosa, VPQ: Volcán Paramillo del Quindío VNS: Volcán Nevado del Santa Isabel, CVNR: Complejo Volcánico Nevado del Ruíz, VCB: Volcán Cerro Bravo, VR: Volcán Romeral, VEE: Volcán el Escondido, VSD: Volcán San Diego. C. Ubicación de los volcanes monogenéticos del CVMVT respecto a la ciudad de Manizales; como referencia el Nevado del Ruiz; los puntos verdes corresponden a los muestreos utilizados en los análisis químicos, y los rojos representan los muestreos realizados para petrografía. 

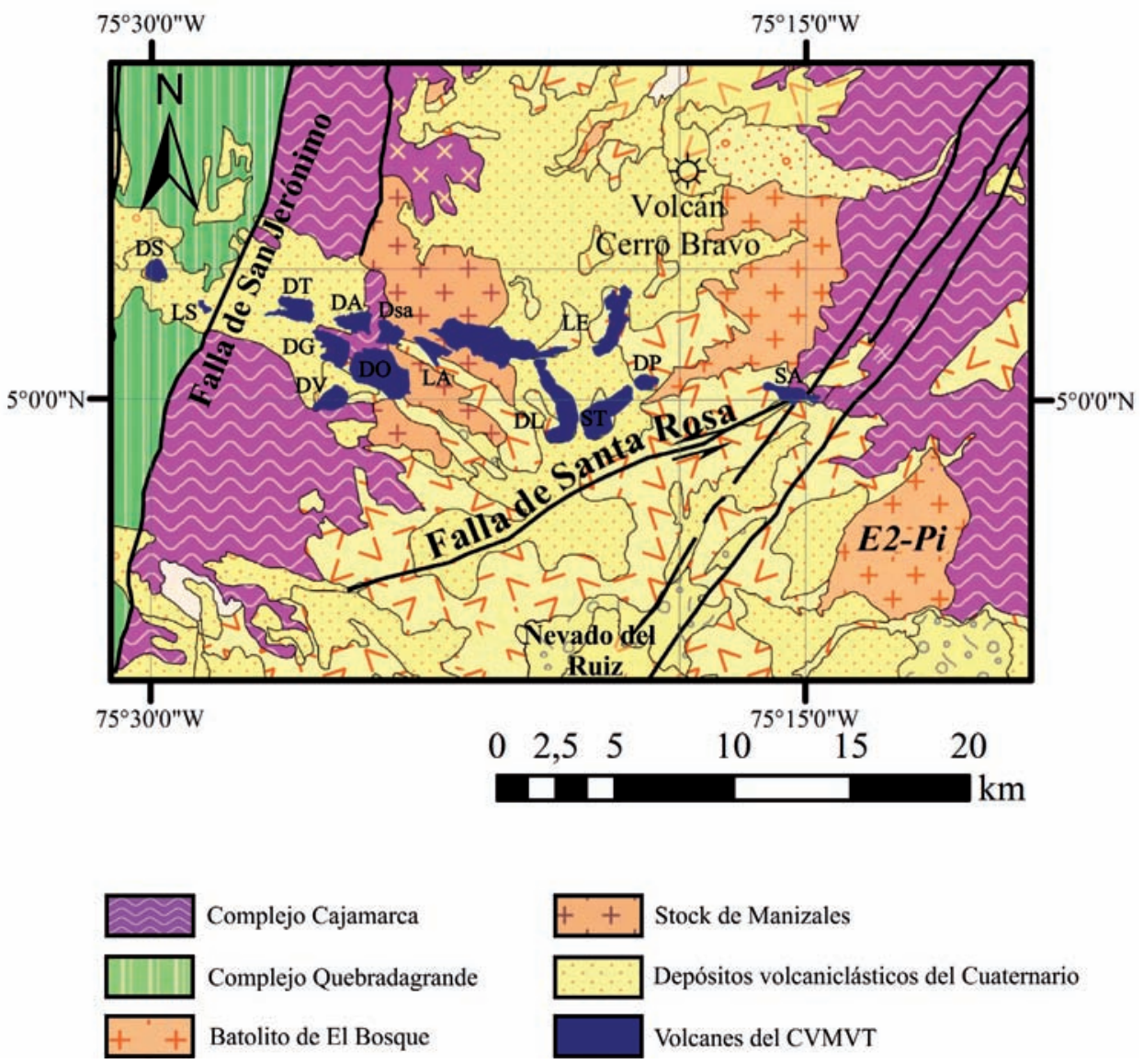

FIGURA 2. Mapa geológico de la zona de estudio. Plancha 5-09 a escala 1:500,000. Los polígonos de color azul representan los volcanes pertenecientes al CVMVT. Adaptado de Gómez et al. (2015). DS: Domo Sancancio, LS: Flujo de lava de Lusitania, DT: Domo Tesorito, DG: Domo Gallinazo, DV: Domo Victoria, DA: Domo Amazonas, DO: Domo La Oliva, DSa: Domo Sabinas, LN: Domo La Negra, LE: Domo La Esperanza, DL: Domo La Laguna, ST: Domo Santana, DP: Domo El Plato y SL: Domo San Luis.

\section{Unidades ígneas y sedimentarias Cenozoicas}

El Stock de Manizales (Mosquera, 1978) o Granodiorita de Manizales (González y Londoño, 2002; FIGURA 2), es un cuerpo ígneo que aflora al $\mathrm{E}$ del municipio de Manizales. En este se determinaron tres tipos de facies: tonalita hornbléndica, tonalita biotítica y granodiorita biotítica (Aguirre y López, 2003). McCourt et al. (1984) reportan edades $\mathrm{K} / \mathrm{Ar}$ en biotita para el stock de $57 \pm 2$ Ma y $56 \pm 3$ Ma. El Batolito de El Bosque (FIGURA 2) corresponde a un cuerpo granítico que aflora en el departamento del Tolima, el cual está compuesto principalmente por cuarzo, plagioclasa, feldespato potásico y biotita. Un análisis geocronológico de K/Ar en biotita sugiere una edad de $49 \pm 1,7$ Ma para este plutón (Vesga y Barrero, 1978).
Ambos cuerpos plutónicos se atribuyen al magmatismo producido en las etapas tempranas de la orogenia Andina (Bustamante et al., 2017). En ambos cuerpos se observan altas relaciones de $\mathrm{Sr} / \mathrm{Y}$, lo cual es característico para todos los plutones de edad Eoceno de la Cordillera Central de Colombia (Bustamante et al., 2017) (FIGURA 2). Según González-Garcia y Jessell (2016), las similitudes composicionales y la localización permiten inferir que ambos cuerpos son parte de un mismo plutón.

\section{Complejo Volcánico Nevado del Ruiz}

El denominado CVNR por Martínez et al. (2004) es típicamente un volcán compuesto (i.e. poligenético) al que se le han atribuido tres períodos eruptivos: Ruiz 
Ancestral (1,8 y 0,8 Ma), Ruiz Antiguo (0,76 a 0,16 Ma) y Ruiz Actual (Thouret et al., 1990; Borrero et al., 2009) (FIGURA 2).

En el período eruptivo Ruiz Ancestral (1,8-0,8 Ma), el volcán emitió flujos de lava de composición andesítica basáltica a andesítica, seguido de una fase destructiva (Thouret et al., 1990; Schaefer, 1995). En el período eruptivo Ruiz Antiguo, el volcán se desarrolló al interior de una presunta caldera y evolucionó entre $\sim 0,76$ y 0,2 Ma, hasta que ocurrió una fase destructiva entre 0,2 y $0,16 \mathrm{Ma}$, en la cual se generaron flujos piroclásticos del orden de 5 a $6 \mathrm{~km}^{3}$; así se sugiere un colapso del edificio volcánico y la formación de una caldera $(5$ a $8 \mathrm{~km}$ de diámetro; Thouret et al., 1990). El período eruptivo Ruiz Actual construido durante los últimos 150.000 años se caracteriza por haber presentado una fase larga de construcción y una fase corta de destrucción, en la cual se emitieron varios flujos de lava y caídas piroclásticas (Thouret et al., 1990). La fase destructiva comenzó hace sólo 11.000 años con emisión de magmas de composición andesítica y dacítica (Thouret et al., 1990).

\section{METODOLOGÍA}

Para la realización de este trabajo se llevó a cabo un análisis composicional que contó con la descripción de secciones delgadas (FIGURA 1C) y la interpretación de 24 análisis químicos reportados previamente (TABLA 1) para 10 de los 14 centros volcánicos que conforman el CVMVT. Los análisis petrográficos incluyeron la identificación de minerales y la descripción de las principales texturas presentes. La interpretación de análisis químicos constó de la diagramación e interpretación de datos compilados desde múltiples trabajos que han reportado análisis para algunos de los centros volcánicos, pero de manera individual y en un contexto de un periodo de evolución del CVNR, más que como un campo monogenético en sí mismo (Ancochea et al., 1991; Toro et al., 2008; Borrero et al., 2009; Rayo, 2012; Martínez et al., 2014). Adicionalmente, los volcanes monogenéticos se compararon composicionalmente entre ellos con el fin de resaltar similitudes y/o diferencias existentes. Estos datos se compararon también con los productos del CVNR con el objetivo de encontrar similitudes y/o diferencias con este volcán poligenético al cual normalmente se ha asociado al CVMVT. Los resultados de análisis químicos se procesaron en el software GCDkit (Janoušek et al., 2006).

\section{RESULTADOS}

\section{Petrografía}

En el CVMVT se encontró una asociación mineralógica común definida por la presencia de piroxeno + anfíbol + plagioclasa, además de características particulares como la presencia de olivino y/o cuarzo. Según sus características mineralógicas, las rocas de los diferentes centros volcánicos se agruparon en los siguientes tipos: 1) Rocas con contenido de olivino; a este grupo pertenecen los domos: Amazonas, La Negra, La Esperanza y Santana; 2) Rocas con contenido de cuarzo; a este grupo pertenecen los domos Oliva y Gallinazo; y 3) Rocas sin contenido de olivino y/o cuarzo; a este grupo pertenecen los demás centros: domos Tesorito, La Laguna, Victoria, Sancancio, El Plato y Flujo de lava de Lusitania.

Las rocas de los centros volcánicos del Grupo 1 presentan una textura general hipocristalina porfirítica, con una masa fundamental compuesta principalmente de vidrio y microlitos de plagioclasa y piroxeno (FIGURA 3A y 3B). Los fenocristales y microfenocristales son principalmente de plagioclasa, oxihornblenda, piroxeno y olivino con formas euhedrales y subhedrales (FIGURA 3C y 3D). También es común encontrar minerales opacos. Como productos de alteración se encuentran clorita y clinozoisita a partir de alteración de oxihornblenda y piroxeno, respectivamente; como textura secundaria se observa textura fluidal dada por plagioclasa (FIGURA 3E y 3F).

Las rocas de los centros volcánicos del Grupo 2 presentan características composicionales disímiles a la asociación general del resto de los centros volcánicos del CVMVT. La textura general es hipocristalina porfirítica, con una masa fundamental compuesta principalmente por microlitos de cuarzo y plagioclasa (FIGURA 4A y 4B). Los fenocristales y microfenocristales son principalmente de plagioclasa, cuarzo, hornblenda, oxihornblenda y piroxeno los cuales presentan formas de subhedral a euhedral con alto grado de alteración. Como minerales de alteración, se observa moscovita, epidota, clinozoisita y carbonatos de calcio (FIGURA 4C y 4D). 
TABLA 1. Recopilación de análisis químicos en roca total por cada volcán del Campo Volcánico Monogenético VillamaríaTermales (CVMVT). La ubicación de las muestras usadas para análisis químico según cada volcán se representa en la FIGURA 1C. (A) Ancochea et al. (1991); (T) Toro et al. (2008); (B) Borrero et al. (2009); (R) Rayo (2012); (M) Martínez et al. (2014).

\begin{tabular}{|c|c|c|c|c|c|c|c|c|c|}
\hline $\begin{array}{l}\text { Autor } \\
\text { Código } \\
\text { Volcán } \\
\end{array}$ & $\begin{array}{c}\text { A } \\
5158 \\
\text { Sancancio } \\
\end{array}$ & $\begin{array}{c}\text { A } \\
5150 \\
\text { Lusitania } \\
\end{array}$ & $\begin{array}{c}\text { A } \\
5151 \\
\text { Tesorito } \\
\end{array}$ & $\begin{array}{c}\text { B } \\
\text { PA-20-3 } \\
\text { Gallinazo } \\
\end{array}$ & $\begin{array}{c}\text { B } \\
\text { PA-21-5 } \\
\text { Gallinazo } \\
\end{array}$ & $\begin{array}{c}\text { B } \\
83 \\
\text { Gallinazo } \\
\end{array}$ & $\begin{array}{c}\mathbf{T} \\
\text { Aa-45 } \\
\text { Gallinazo } \\
\end{array}$ & $\begin{array}{c}\mathbf{T} \\
\text { Aa-45a } \\
\text { Gallinazo }\end{array}$ & $\begin{array}{c}\text { A } \\
5159 \\
\text { Gallinazo } \\
\end{array}$ \\
\hline \multicolumn{10}{|l|}{ wt,\% } \\
\hline $\mathrm{SiO}_{2}$ & 59,57 & 58,86 & 62,8 & 62,46 & 60,92 & 63,53 & 65,77 & 65,72 & 63,55 \\
\hline $\mathrm{TiO}_{2}$ & 0,65 & 0,82 & 0,7 & 0,41 & 0,57 & 0,54 & 0,55 & 0,54 & 0,66 \\
\hline $\mathrm{Al}_{2} \mathrm{O}_{3}$ & 16,21 & 17,15 & 15,62 & 15,58 & 16,34 & 16,83 & 16,22 & 16,23 & 15,87 \\
\hline $\mathrm{Fe}_{2} \mathrm{O}_{3}$ & 2,43 & 3,23 & 2,55 & 4,38 & 4,56 & 4,56 & 4,68 & 4,77 & 3,77 \\
\hline $\mathrm{FeO}$ & 2,41 & 1,89 & 2,32 & - & - & - & - & - & - \\
\hline $\mathrm{MnO}$ & 0,09 & 0,12 & 0,08 & 0,13 & 0,18 & 0,06 & 0,07 & 0,07 & 0,07 \\
\hline $\mathrm{MgO}$ & 5,04 & 4,19 & 2,82 & 1,19 & 1,85 & 1,03 & 1,93 & 1,95 & 2,55 \\
\hline $\mathrm{CaO}$ & 6,2 & 5,48 & 4,68 & 3,54 & 3,37 & 1,55 & 0,46 & 0,47 & 3,65 \\
\hline $\mathrm{Na}_{2} \mathrm{O}$ & 3,98 & 3,81 & 4,14 & 3,04 & 3,67 & 0,77 & 2,77 & 2,79 & 3,9 \\
\hline $\mathrm{K}_{2} \mathrm{O}$ & 1,36 & 1,05 & 2,47 & 2,35 & 2,9 & 3,15 & 1,91 & 1,9 & 2,89 \\
\hline $\mathrm{P}_{2} \mathrm{O}_{5}$ & 0,2 & 0,2 & 0,24 & 0,19 & 0,26 & 0,24 & 0,23 & 0,24 & 0,22 \\
\hline $\mathrm{Cr}_{2} \mathrm{O}_{3}$ & - & - & - & 0 & 0 & 0,01 & - & - & - \\
\hline LOI\% & 1,01 & 1,48 & 0,98 & 9,46 & 6,33 & 10,69 & 5,1 & 5 & 0,84 \\
\hline TOTAL & 100,17 & 100,12 & 99,35 & 102,29 & 100,49 & 102,49 & 99,69 & 99,68 & 99,47 \\
\hline Ва & 1061 & 937 & 1361 & 945,9 & 1547,6 & 1027,5 & 1296 & 1336 & 1479 \\
\hline $\mathrm{Rb}$ & 40 & 33 & 74 & 82,3 & 74,7 & 89,9 & 49,5 & 50,7 & 99 \\
\hline Th & 7 & 3 & 18 & 3,9 & 7,2 & 5 & 5,2 & 6,5 & 13 \\
\hline $\mathrm{Nb}$ & 8 & 8 & 8 & 5,3 & 6,5 & 5,5 & 5,6 & 5,9 & 10 \\
\hline $\mathrm{Sr}$ & 621 & 524 & 768 & 367,2 & 472,5 & 94,4 & 348 & 360 & 586 \\
\hline $\mathrm{Zr}$ & 100 & 94 & 166 & 101,6 & 124,8 & 103 & 114,5 & 117,6 & 210 \\
\hline $\mathrm{Y}$ & 18 & 22 & 17 & 13,1 & 16,7 & 27 & 15,1 & 15,9 & 18 \\
\hline $\mathrm{Cr}$ & - & - & - & - & - & - & 0,01 & 0,01 & - \\
\hline $\mathrm{V}$ & - & - & - & 74 & 100 & 95 & 106 & 113 & - \\
\hline $\mathrm{Ni}$ & 106 & 22 & 47 & 5,6 & 6,8 & 20,2 & 16,2 & 15,7 & 44 \\
\hline Co & - & - & - & 8,3 & 7 & 9,4 & 10,1 & 10,5 & - \\
\hline Sc & - & - & - & 8 & 11 & 12 & 13 & 13 & - \\
\hline $\mathrm{Pb}$ & - & - & - & 14,8 & 13,2 & 29,8 & - & - & - \\
\hline $\mathrm{Cu}$ & - & - & - & 3,6 & 7,9 & 20,8 & 18,3 & 17,8 & - \\
\hline $\mathrm{Zn}$ & - & - & - & 39 & 72 & 764 & - & - & - \\
\hline Cs & - & - & - & 3 & 3,5 & 7,4 & 2,5 & 2,6 & - \\
\hline $\mathrm{Be}$ & - & - & - & 1 & 1 & 1 & - & - & - \\
\hline $\mathrm{Ga}$ & - & - & - & 18,5 & 19,1 & 17,5 & - & - & - \\
\hline Mo & - & - & - & 0,7 & 1 & 0,5 & - & - & - \\
\hline Sn & - & - & - & 1 & 1 & 2 & 2 & 2 & - \\
\hline $\mathrm{Tl}$ & - & - & - & 0,2 & 0,1 & 0,2 & - & - & - \\
\hline $\mathrm{Ta}$ & - & - & - & 0,3 & 0,4 & 0,4 & 0,4 & 0,5 & - \\
\hline $\mathrm{Hf}$ & - & - & - & 3,4 & 3,8 & 3 & 3,5 & 3,6 & - \\
\hline $\mathrm{U}$ & - & - & - & 1,9 & 2 & 2 & - & - & - \\
\hline $\mathrm{La}$ & 12,81 & 15 & 25 & 14,5 & 20,8 & 18 & 17,4 & 18,6 & 33 \\
\hline $\mathrm{Ce}$ & 31,56 & 44 & 47 & 30,3 & 41,5 & 34,8 & 37,1 & 39,1 & 64 \\
\hline $\operatorname{Pr}$ & - & - & - & 3,84 & 5,23 & 4,43 & 4,78 & 4,97 & - \\
\hline $\mathrm{Nd}$ & 14,61 & - & - & 15,5 & 21,2 & 18,7 & 20,9 & 20,9 & - \\
\hline $\mathrm{Sm}$ & 3,57 & - & - & 2,96 & 3,88 & 3,66 & 3,86 & 3,97 & - \\
\hline $\mathrm{Eu}$ & 0,96 & - & - & 0,82 & 1 & 1,22 & 1,07 & 1,1 & - \\
\hline Gd & - & - & - & 2,9 & 3,43 & & 3,13 & 3,35 & - \\
\hline $\mathrm{Tb}$ & - & - & - & 0,43 & 0,55 & 0,62 & 0,56 & 0,58 & - \\
\hline Dy & 2,54 & - & - & 2,44 & 2,87 & 3,55 & 3,12 & 3,06 & - \\
\hline Но & - & - & - & 0,46 & 0,52 & 0,73 & 0,52 & 0,54 & - \\
\hline Er & 1,34 & - & - & 1,21 & 1,47 & 2,11 & 1,41 & 1,48 & - \\
\hline $\mathrm{Tm}$ & - & - & - & 0,19 & 0,24 & 0,33 & 0,21 & 0,25 & - \\
\hline $\mathrm{Yb}$ & - & 1,28 & - & 1,16 & 1,51 & 1,93 & 1,38 & 1,5 & - \\
\hline $\mathrm{Lu}$ & - & 0,24 & - & 0,2 & 0,24 & 0,31 & 0,24 & 0,24 & - \\
\hline
\end{tabular}


Continuación TABLA 1

\begin{tabular}{|c|c|c|c|c|c|c|c|c|}
\hline Autor & B & B & B & B & B & B & A & A \\
\hline Código & Pa-15g & Pa-31a & Pa-32-5 & Pa-8a-1 & Pa-9-1 & Pa-10-2 & 5160 & 5161 \\
\hline Volcán & Amazonas & Amazonas & Amazonas & La Oliva & La Oliva & La Oliva & La Negra & La Negra \\
\hline $\mathrm{SiO}_{2}$ & 67,88 & 67,91 & 62,74 & 64,29 & 63,86 & 63,77 & 61,88 & 61,59 \\
\hline $\mathrm{TiO}_{2}$ & 0,45 & 0,38 & 0,64 & 0,57 & 0,55 & 0,46 & 0,63 & 0,79 \\
\hline $\mathrm{Al}_{2} \mathrm{O}_{3}$ & 14,8 & 14,18 & 16,2 & 16,7 & 16,45 & 15,91 & 16,4 & 16,19 \\
\hline $\mathrm{Fe}_{2} \mathrm{O}_{3}$ & 3,56 & 2,85 & 5,02 & 4,63 & 5,4 & 5,09 & 3 & 3,09 \\
\hline $\mathrm{MnO}$ & 0,04 & 0,05 & 0,07 & 0,03 & 0,04 & 0,03 & 0,06 & 0,09 \\
\hline $\mathrm{MgO}$ & 1,25 & 1,38 & 2,45 & 1,87 & 1,61 & 1,38 & 2,96 & 3,46 \\
\hline $\mathrm{CaO}$ & 2,61 & 2,56 & 4,67 & 1,19 & 1,76 & 1,99 & 4,71 & 5,24 \\
\hline $\mathrm{Na}_{2} \mathrm{O}$ & 3,71 & 3,65 & 4,38 & 3,16 & 3,51 & 4,64 & 4,38 & 4,29 \\
\hline $\mathrm{K}_{2} \mathrm{O}$ & 3,78 & 4,01 & 2,33 & 1,9 & 1,19 & 1,33 & 2,35 & 2,26 \\
\hline $\mathrm{P}_{2} \mathrm{O}_{5}$ & 0,12 & 0,12 & 0,23 & 0,15 & 0,24 & 0,2 & 0,3 & 0,3 \\
\hline $\mathrm{Cr}_{2} \mathrm{O}_{3}$ & 0,01 & 0,01 & 0,01 & - & - & - & - & - \\
\hline LOI\% & 1,7 & 2,69 & 0,96 & 7,42 & 8,2 & 7,17 & 0,99 & 0,47 \\
\hline TOTAL & 99,54 & 99,49 & 99,19 & 101,45 & 102,27 & 101,46 & 99,55 & 100,18 \\
\hline $\mathrm{Ba}$ & 1273,5 & 1133,9 & 1271,3 & 980,4 & 854,4 & 334 & 1396 & 1324 \\
\hline $\mathrm{Rb}$ & 131,7 & 149,3 & 64,5 & 72,1 & 45,7 & 61,7 & 68 & 64 \\
\hline Th & 26,6 & 32,1 & 11,5 & 8,4 & 4,7 & 4,8 & 10 & 7 \\
\hline $\mathrm{Nb}$ & 9,6 & 10 & 6,9 & 6,3 & 5,8 & 5,8 & 7 & 11 \\
\hline $\mathrm{Sr}$ & 437,5 & 425,1 & 739,4 & 448 & 438,1 & 351,5 & 808 & 825 \\
\hline $\mathrm{Zr}$ & 145,6 & 150,8 & 125,6 & 116,8 & 119,7 & 114,9 & 153 & 164 \\
\hline $\mathrm{Y}$ & 12,6 & 14,3 & 12,4 & 13,6 & 12 & 11,2 & 18 & 18 \\
\hline V & 70 & 58 & 115 & 117 & 86 & 79 & - & - \\
\hline $\mathrm{Ni}$ & 11,4 & 8,2 & 14,2 & 7,5 & 5,8 & 8,7 & 39 & 30 \\
\hline Co & 8,4 & 7,1 & 13,5 & 8,4 & 14,6 & 7,4 & - & - \\
\hline Sc & 7 & 6 & 11 & 11 & 9 & 8 & - & - \\
\hline $\mathrm{Pb}$ & 5,2 & 1,3 & 1,5 & 18,6 & 9,9 & 13,7 & - & - \\
\hline $\mathrm{Cu}$ & 20,7 & 14,4 & 10 & 140,8 & 225,1 & 75 & - & - \\
\hline $\mathrm{Zn}$ & 47 & 17 & 43 & 2 & 62 & 83 & - & - \\
\hline Cs & 3,7 & 10,5 & 2,1 & 6,5 & 11,3 & 6,3 & - & - \\
\hline $\mathrm{Be}$ & 2 & 2 & 1 & 1 & 1 & 1 & - & - \\
\hline $\mathrm{Ga}$ & 19,5 & 18,8 & 21 & 21,6 & 17,9 & 20,1 & - & - \\
\hline Mo & 1,6 & 0,5 & 1,7 & 5,3 & 2,2 & 0,5 & - & - \\
\hline Sn & 1 & 2 & 1 & 3 & 3 & 1 & - & - \\
\hline $\mathrm{Tl}$ & 0,1 & 0,1 & 0,1 & 0,2 & 0,1 & 0,2 & - & - \\
\hline Ta & 0,9 & 1,1 & 0,5 & 0,4 & 0,4 & 0,4 & - & - \\
\hline Hf & 5,1 & 5,1 & 3,9 & 3,2 & 3,6 & 3,2 & - & - \\
\hline $\mathrm{U}$ & 12,7 & 14,3 & 4,4 & 2,7 & 2 & 2,2 & - & - \\
\hline $\mathrm{La}$ & 23,5 & 26,7 & 20,8 & 29,7 & 17 & 18,9 & 33 & 27 \\
\hline Ce & 43,5 & 49,1 & 40,4 & 57,6 & 36 & 38,2 & 53 & 49 \\
\hline $\operatorname{Pr}$ & 5,25 & 5,94 & 5,16 & 7,11 & 4,62 & 4,7 & - & - \\
\hline $\mathrm{Nd}$ & 20,1 & 21,9 & 20,5 & 28,1 & 19,7 & 18,5 & - & - \\
\hline $\mathrm{Sm}$ & 3,5 & 3,52 & 3,83 & 5,18 & 3,63 & 3,19 & - & - \\
\hline $\mathrm{Eu}$ & 0,74 & 0,71 & 1 & 1,26 & 0,87 & 0,84 & - & - \\
\hline Gd & 2,74 & - & - & 2,39 & 2,43 & 3,05 & - & - \\
\hline $\mathrm{Tb}$ & 0,43 & 0,46 & 0,48 & 0,59 & 0,45 & 0,4 & - & - \\
\hline Dy & 2,27 & 2,26 & 2,34 & 2,82 & 2,32 & 1,87 & - & - \\
\hline Но & 0,4 & 0,42 & 0,42 & 0,48 & 0,4 & 0,34 & - & - \\
\hline Er & 1,16 & 1,22 & 1,03 & 1,29 & 1,23 & 1 & - & - \\
\hline $\mathrm{Tm}$ & 0,19 & 0,19 & 0,19 & 0,22 & 0,19 & 0,17 & - & - \\
\hline $\mathrm{Yb}$ & 1,2 & 1,34 & 1,08 & 1,37 & 1,15 & 0,94 & - & - \\
\hline $\mathrm{Lu}$ & 0,21 & 0,23 & 0,16 & 0,23 & 0,2 & 0,16 & - & - \\
\hline
\end{tabular}


Continuación TABLA 1

\begin{tabular}{|c|c|c|c|c|c|c|c|}
\hline Autor & $\mathbf{T}$ & A & M & M & $\mathbf{R}$ & $\mathbf{R}$ & M \\
\hline Código & Aa-79 & 5157 & 5004932 & 5004913 & GNR-LR-210 & GNR-LR-203 & 5004914 \\
\hline Volcán & La Negra & La Negra & La Esperanza & La Laguna & La Laguna & Santana & Santana \\
\hline $\mathrm{SiO}_{2}$ & 61,46 & 63,23 & 55,95 & 59,78 & 58,77 & 57,72 & 60,88 \\
\hline $\mathrm{TiO}_{2}$ & 0,69 & 0,74 & 0,77 & 0,74 & 0,73 & 0,73 & 0,70 \\
\hline $\mathrm{Al}_{2} \mathrm{O}_{3}$ & 15,82 & 15,58 & 14,81 & 16,10 & 16,42 & 16,68 & 16,10 \\
\hline $\mathrm{Fe}_{2} \mathrm{O}_{3}$ & 5,59 & 2,67 & 7,58 & 6,52 & 6,74 & 6,61 & 6,17 \\
\hline $\mathrm{FeO}$ & - & - & 4,63 & 3,00 & - & - & 2,19 \\
\hline $\mathrm{MnO}$ & 0,07 & 0,07 & - & - & 0,11 & 0,11 & - \\
\hline $\mathrm{MgO}$ & 3,25 & 2,95 & 8,50 & 4,35 & 4,37 & 4,29 & 3,96 \\
\hline $\mathrm{CaO}$ & 4,94 & 4,55 & 7,55 & 6,70 & 6,72 & 6,26 & 6,37 \\
\hline $\mathrm{Na}_{2} \mathrm{O}$ & 3,95 & 4 & 3,12 & 3,41 & 3,58 & 3,56 & 3,54 \\
\hline $\mathrm{K}_{2} \mathrm{O}$ & 2,15 & 2,72 & 1,15 & 1,29 & 1,28 & 1,33 & 1,33 \\
\hline $\mathrm{P}_{2} \mathrm{O}_{5}$ & 0,25 & 0,24 & 0,19 & 0,18 & 0,16 & 0,2 & 0,19 \\
\hline $\mathrm{Cr}_{2} \mathrm{O}_{3}$ & - & - & - & - & 0,011 & 0,011 & - \\
\hline LOI\% & 1,6 & 1 & $<0,10$ & $0,64-$ & 0,8 & 2,3 & $0,46-$ \\
\hline TOTAL & 99,77 & 100,07 & - & - & 99,74 & 99,75 & - \\
\hline $\mathrm{Ba}$ & 1225 & 1319 & 1015 & 1001 & 712 & 715 & 1106 \\
\hline $\mathrm{Rb}$ & 63,4 & 88 & 24,5 - & 31,4 & 23 & 23,8 & $32,1-$ \\
\hline Th & 8,5 & 15 & 2,5 & 4 & 3,4 & 3,8 & 4,1 \\
\hline $\mathrm{Nb}$ & 6,8 & - & - & - & 3,9 & 4,2 & - \\
\hline $\mathrm{Sr}$ & 693 & 697 & 519 & 677 & 612,8 & 558,5 & 636 \\
\hline $\mathrm{Zr}$ & 133,6 & 186 & $<130$ & $<130$ & 80,1 & 75,5 & $<130$ \\
\hline $\mathrm{Y}$ & 14,1 & 16 & 15,7 & 14,8 & 12,3 & 12,9 & 15,4 \\
\hline $\mathrm{Cr}$ & 0,02 & - & 523 & 104 & - & - & 96 \\
\hline $\mathrm{V}$ & 127 & - & 162 & 179 & 184 & 175 & 168 \\
\hline $\mathrm{Ni}$ & 14,3 & 38 & 212 & 13 & 4,9 & 4,8 & 11 \\
\hline Co & 16,5 & - & - & - & 15,3 & 14 & - \\
\hline Sc & 14 & - & - & - & 0,9 & 0,6 & - \\
\hline $\mathrm{Pb}$ & - & - & 9,0 & 10 & 1 & 1,3 & 9,3 \\
\hline $\mathrm{Cu}$ & 13,5 & - & 53 & 16 & 212,2 & 9,9 & 18 \\
\hline $\mathrm{Zn}$ & - & - & 91 & 90 & 26 & 34 & 83 \\
\hline Cs & 1,7 & - & - & - & - & - & - \\
\hline $\mathrm{Be}$ & - & - & 1 & 1,06 & $<1$ & $<1$ & 1,24 \\
\hline $\mathrm{Ga}$ & - & - & 20 & 20,8 & 17,8 & 18,1 & 21,2 \\
\hline Mo & - & - & - & - & 0,5 & 0,4 & - \\
\hline Sn & 1 & - & - & - & 1 & $<1$ & - \\
\hline $\mathrm{Tl}$ & - & - & 0,08 & 0,02 & $<0,1$ & $<0,1$ & 0,15 \\
\hline Тa & 0,5 & - & - & - & 0,3 & 0,3 & - \\
\hline Hf & 4,1 & - & - & - & 2,5 & 2,3 & - \\
\hline $\mathrm{U}$ & - & - & 1,27 & 1,36 & 1,2 & 1,2 & 1,49 \\
\hline $\mathrm{La}$ & 21 & 16 & 15 & 15 & 11,6 & 11,7 & 17 \\
\hline Ce & 40,2 & 61 & 30 & 32 & 24,1 & 24,5 & 34 \\
\hline $\operatorname{Pr}$ & 5,64 & - & 4,1 & 3,9 & 2,96 & 3,1 & 4,2 \\
\hline $\mathrm{Nd}$ & 22 & - & 17,9 & 16,9 & 12,9 & 13,2 & 18,1 \\
\hline $\mathrm{Sm}$ & 4,09 & - & 4,1 & 3,7 & 279,79 & 289 & 3,9 \\
\hline $\mathrm{Eu}$ & 1,06 & - & 1,5 & 1,44 & 0,9 & 0,9 & 1,43 \\
\hline $\mathrm{Gd}$ & 3,23 & - & 3,85 & 3,51 & 2,76 & 2,83 & 3,7 \\
\hline $\mathrm{Tb}$ & 0,53 & - & 0,6 & 0,54 & 0,44 & 0,44 & 0,52 \\
\hline Dy & 2,8 & - & 3,13 & 2,93 & 2,21 & 2,57 & 3,02 \\
\hline Ho & 0,48 & - & 0,6 & 0,6 & 0,48 & 0,48 & 0,61 \\
\hline Er & 1,25 & - & 1,74 & 1,77 & 1,36 & 1,39 & 1,79 \\
\hline $\mathrm{Tm}$ & 0,21 & - & 0,23 & 0,25 & 0,2 & 0,2 & 0,25 \\
\hline $\mathrm{Yb}$ & 1,22 & - & 1,52 & 1,67 & 1,28 & 1,35 & 1,69 \\
\hline $\mathrm{Lu}$ & 0,19 & - & 0,22 & 0,25 & 0,19 & 0,2 & 0,25 \\
\hline
\end{tabular}



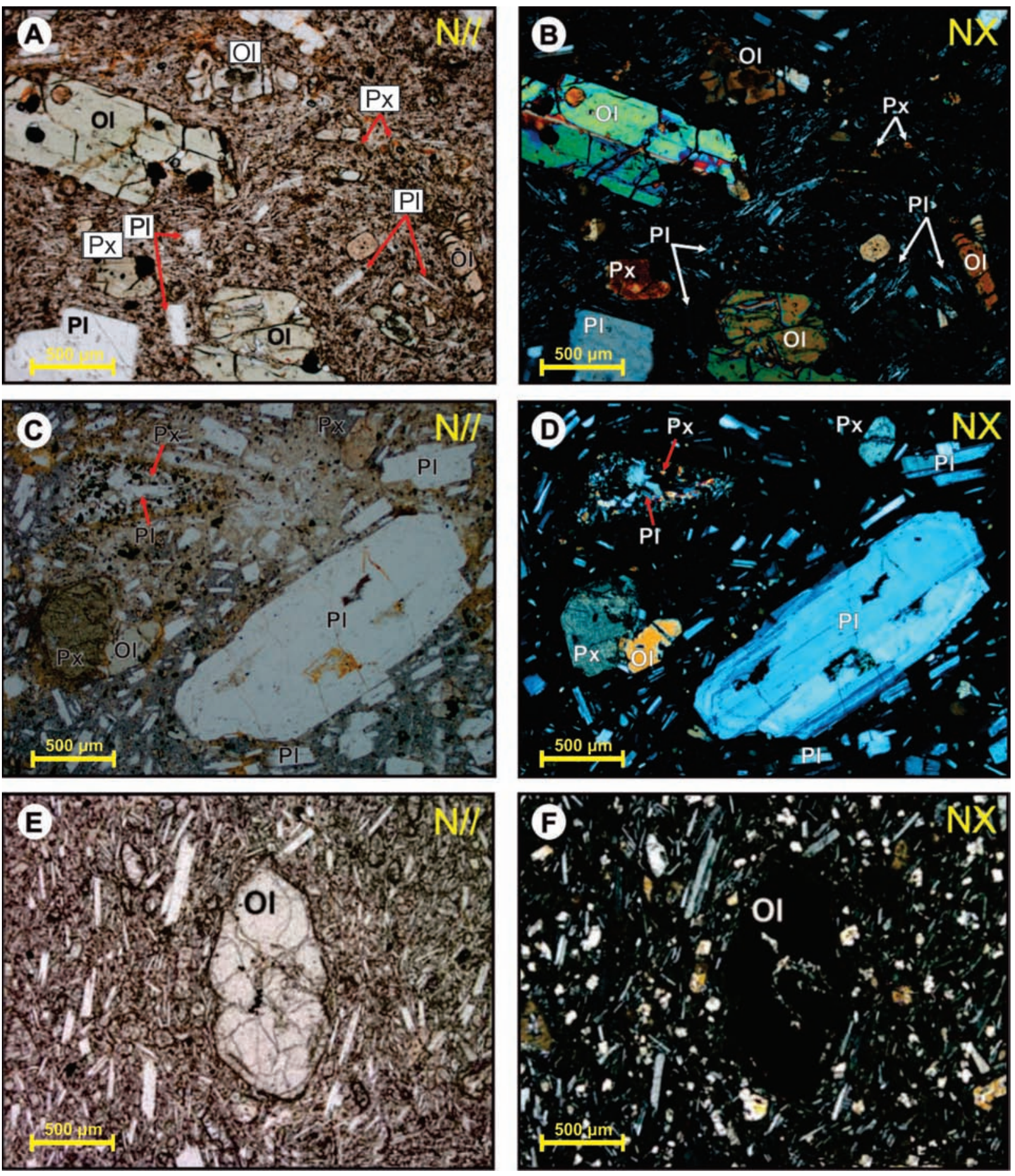

FIGURA 3. Fotomicrografía de las rocas volcánicas de los domos La Negra, Amazonas y La Esperanza. N//: nícoles paralelos; NX: nícoles cruzados. A. y B. Fenocristales de olivino y plagioclasa; también se observan microfenocristales de piroxeno y plagioclasa en la matriz. C. y D. Fenocristal de plagioclasa y microfenocristales de piroxeno y olivino; además se observa textura cumulo porfirítica de piroxeno y plagioclasa. E. y F. Microcristales de olivino, plagioclasa y piroxeno en matriz microcristalina y vítrea. Modificada de Martínez et al. (2014). Abreviaciones: (Pl): Plagioclasa, (Px): Piroxeno, (Ol): Olivino. 

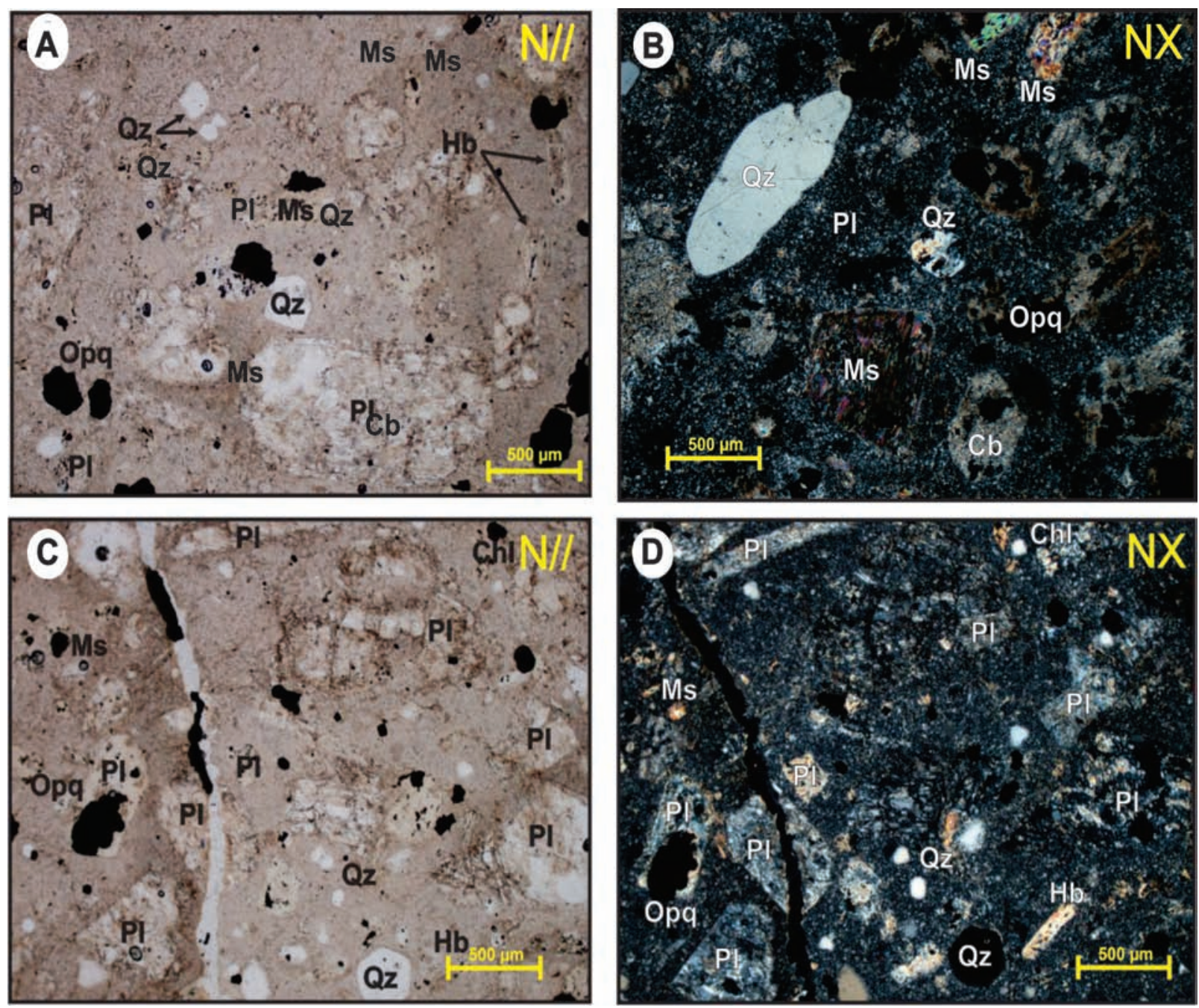

FIGURA 4. Fotomicrografía de las rocas de los domos Gallinazo y La Oliva. N//: nícoles paralelos; NX: nícoles cruzados. A. y B. Microfenocristales de cuarzo, moscovita y carbonatos embebidos en una matriz criptocristalina y microcristalina. La plagioclasa se encuentra sausuritizada y algunos cristales también se encuentran reemplazados por carbonatos. C. y D. Microfenocristales de plagioclasa, cuarzo y hornblenda, acompañados de sausurita y carbonatos como productos de alteración de plagioclasa, y clorita como alteración de la hornblenda. Abreviaciones: (Pl): Plagioclasa, (Hb): Hornblenda, (Qz): Cuarzo, (Ms): Moscovita, (Opq): Minerales opacos, (Cb): Carbonatos (Chl): Clorita.

Las rocas de los centros volcánicos del Grupo 3 tienen una textura general hipocristalina porfirítica, a excepción del Domo La Laguna que presenta una textura general hipohialina porfitírica. La masa fundamental está compuesta principalmente por microlitos subhedrales de plagioclasa, piroxeno y oxihornblenda. Los fenocristales presentes son: plagioclasa y piroxeno euhedrales, y oxihornblenda subhedrales (FIGURA 5A y 5B). También se encuentran minerales opacos y clinozoisita como producto de alteración a partir del piroxeno. Adicionalmente, en las muestras de los domos La Laguna y El Plato, se observan cristales de feldespato potásico y cuarzo, respectivamente, los cuales presentan bordes de reacción (FIGURA 5C, 5D, 5E y 5F). 

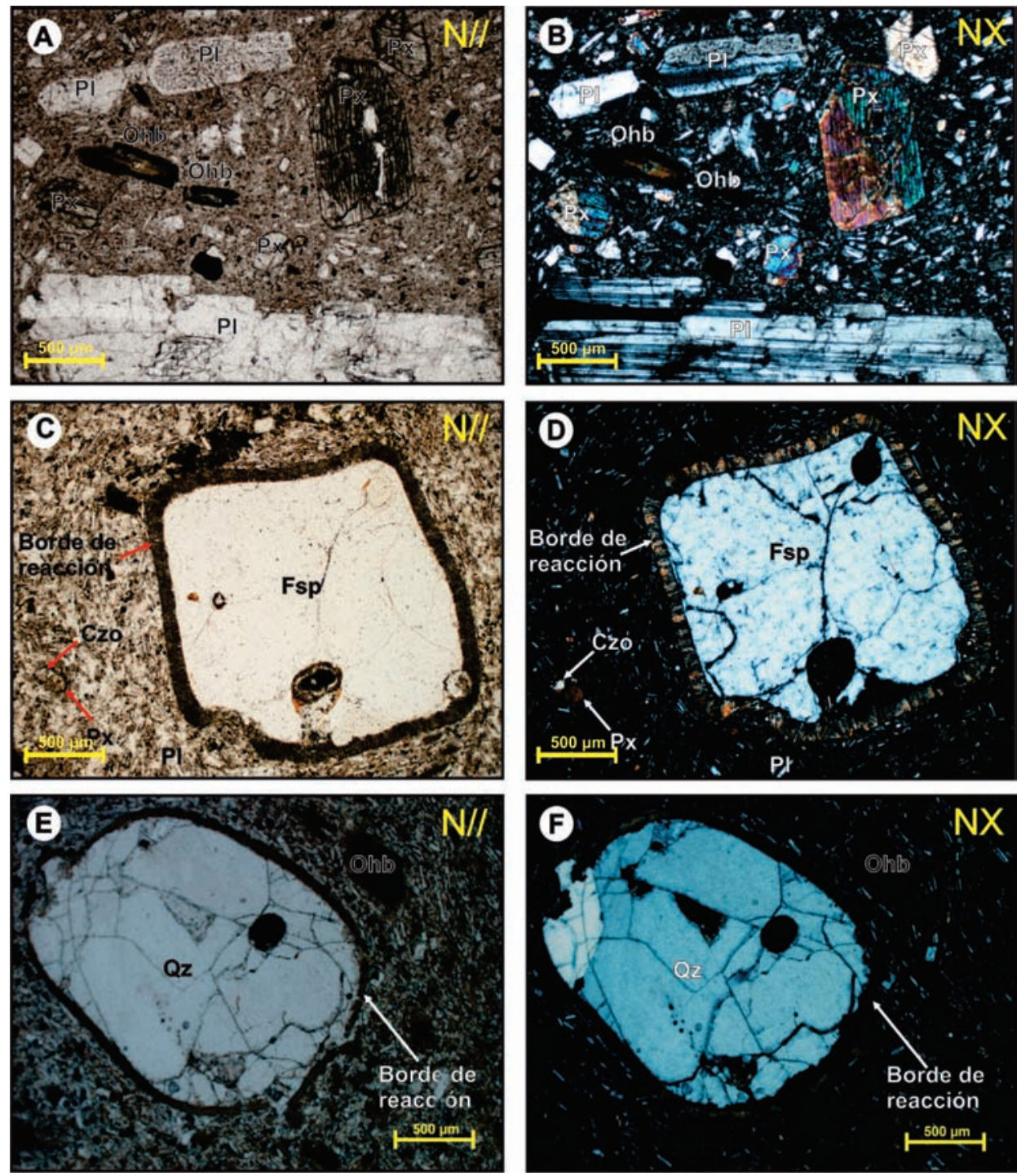

FIGURA 5. Fotomicrografía de las rocas de los domos Victoria, La Laguna y El Plato. N//: nícoles paralelos; NX: nícoles cruzados. A. y B. Fenocristales de plagioclasa y microfenocristales de piroxeno y oxihornblenda embebidos en matriz vítrea microcristalina. C. y D. Xenocristal de feldespato potásico con borde de reacción; también se observan en la matriz microcristales de clinozoisita y piroxeno. E. y F. Xenocristal de cuarzo con borde de reacción. Abreviaciones: (Pl): Plagioclasa, (Ohb): Oxihornblenda, (Px): Piroxeno, (Fsp): Feldespato potásico, (Qz): Cuarzo.

\section{Geoquímica}

Como se observa en el diagrama TAS (Le Bas et al., 1986; FIGURA 6A), 10 de los volcanes pertenecientes al CVMVT tienen una composición que varía entre andesita y dacita, mientras que los domos La Esperanza y Amazonas presentan una composición de andesita basáltica y riolita, respectivamente.

Según el diagrama AFM (Irvine y Baragar, 1971), las muestras analizadas se encuentran en el campo de la serie calcoalcalina (FIGURA 6B). Las muestras de los domos La Laguna, La Esperanza, Santana, La Negra,
Gallinazo, Sancancio y La Oliva se ubican a su vez, dentro del campo de la serie calcoalcalina media en potasio, mientras que dos muestras analizadas del Domo Amazonas, tres del Domo Gallinazo y una del Domo La Negra, se ubican dentro de la serie calcoalcalina rica en potasio (FIGURA 6C).

Según los diagramas tipo Harker para óxidos mayores (FIGURA 7), se puede observar una tendencia general positiva de $\mathrm{K}_{2} \mathrm{O}$, y negativa de $\mathrm{TiO}_{2}$, $\mathrm{CaO}, \mathrm{MgO}$ y $\mathrm{FeO}$, respecto al aumento de $\mathrm{SiO}_{2}$, mientras que $\mathrm{Al}_{2} \mathrm{O}_{3}, \mathrm{Na}_{2} \mathrm{O}$ y $\mathrm{P}_{2} \mathrm{O}_{5}$ no muestran ninguna tendencia en particular. 


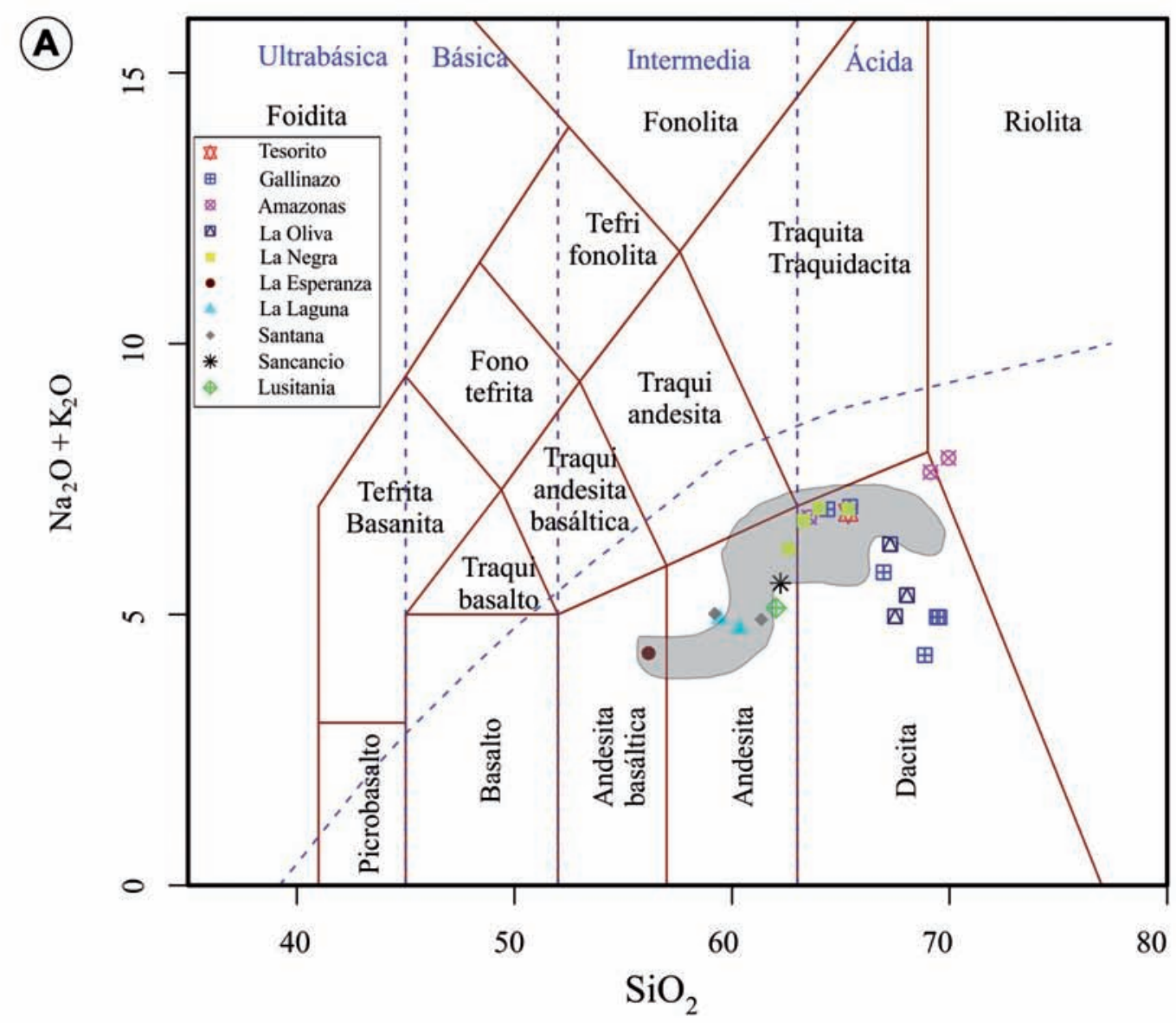

(B)

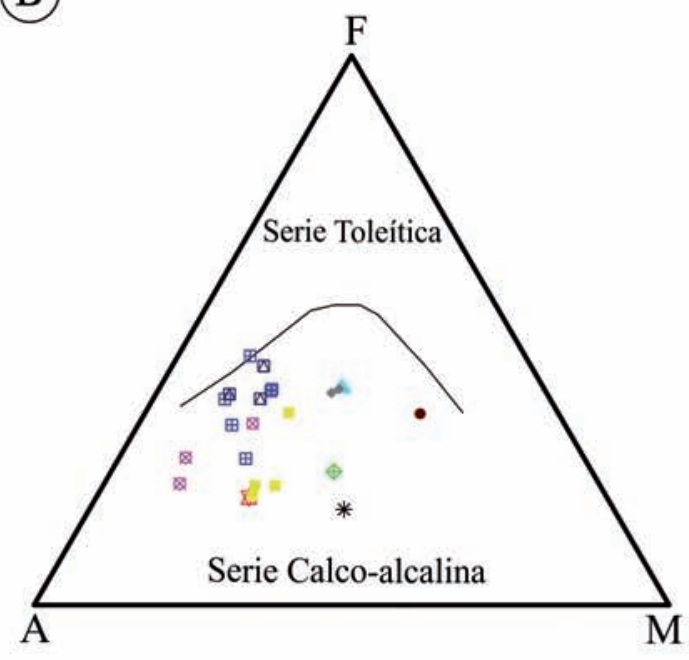

(C)

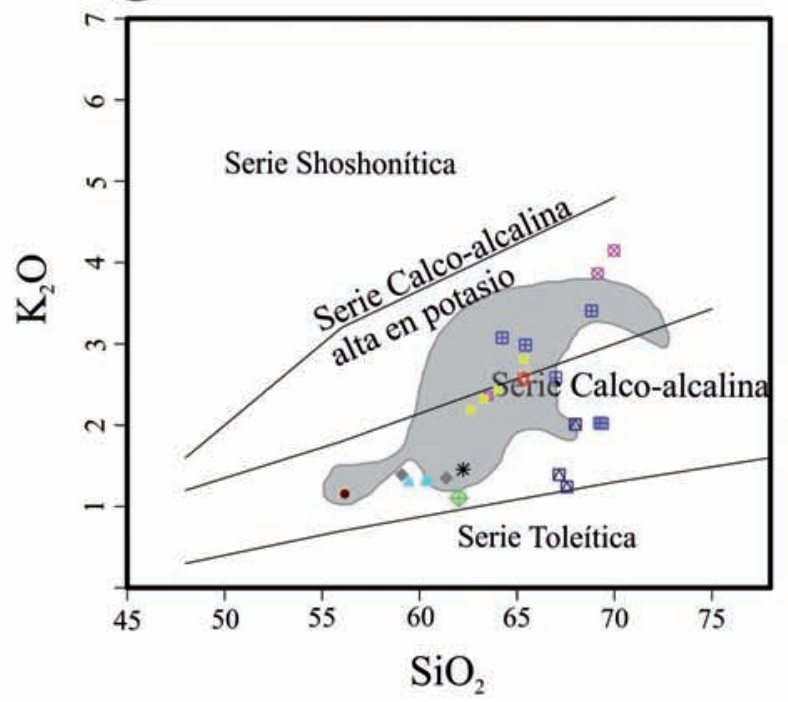

FIGURA 6. Diagramas de clasificación geoquímica para los volcanes del Campo Volcánico Monogenético Villamaría-Termales (CVMVT). A. Diagrama de variación de álcalis vs sílice (TAS) de Le Bas et al. (1986). B. Diagrama AFM de Irvine y Baragar (1971). C. Diagrama de variación $\mathrm{K}_{2} \mathrm{O}$ vs $\mathrm{SiO}_{2}$ de Peccerillo y Taylor (1976). El sombreado gris en la FIGURA 6A y 6C, corresponde a la tendencia composicional de varios productos del Complejo Volcánico Nevado del Ruiz, reportados por Martínez et al. (2014). 
Específicamente, el Domo La Esperanza se destaca por tener un mayor contenido de $\mathrm{MgO}, \mathrm{FeO}$ y $\mathrm{CaO}$, así como por tener un menor contenido de $\mathrm{Al}_{2} \mathrm{O}_{3}$ en comparación con los demás volcanes. El Domo Amazonas se destaca por presentar un bajo contenido de $\mathrm{Al}_{2} \mathrm{O}_{3}$ y $\mathrm{TiO}_{2}$, y un alto contenido de $\mathrm{K}_{2} \mathrm{O}$, mientras
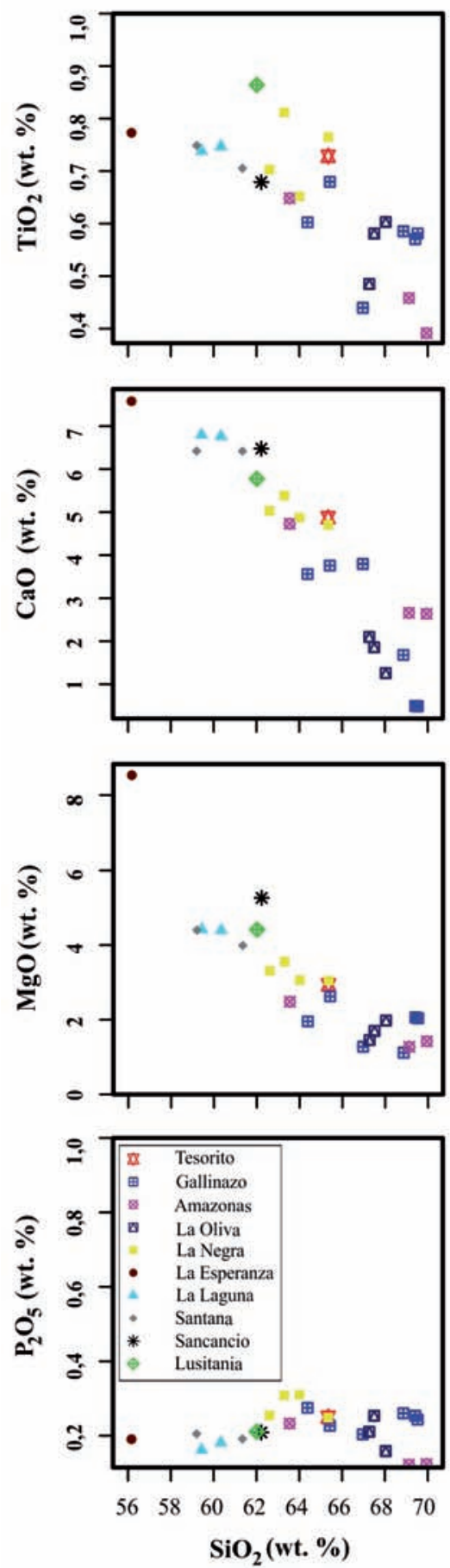

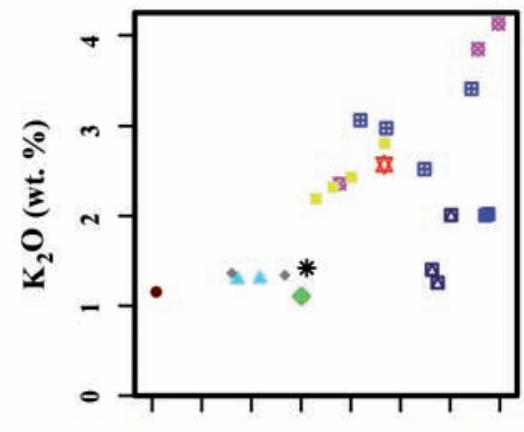

que los domos Sancancio, Tesorito y La Negra por presentar un bajo contenido de FeO. Una muestra del Domo Gallinazo se destaca por presentar un bajo contenido de $\mathrm{Na}_{2} \mathrm{O}$, mientras que una muestra del Domo La Oliva por presentar un alto contenido.
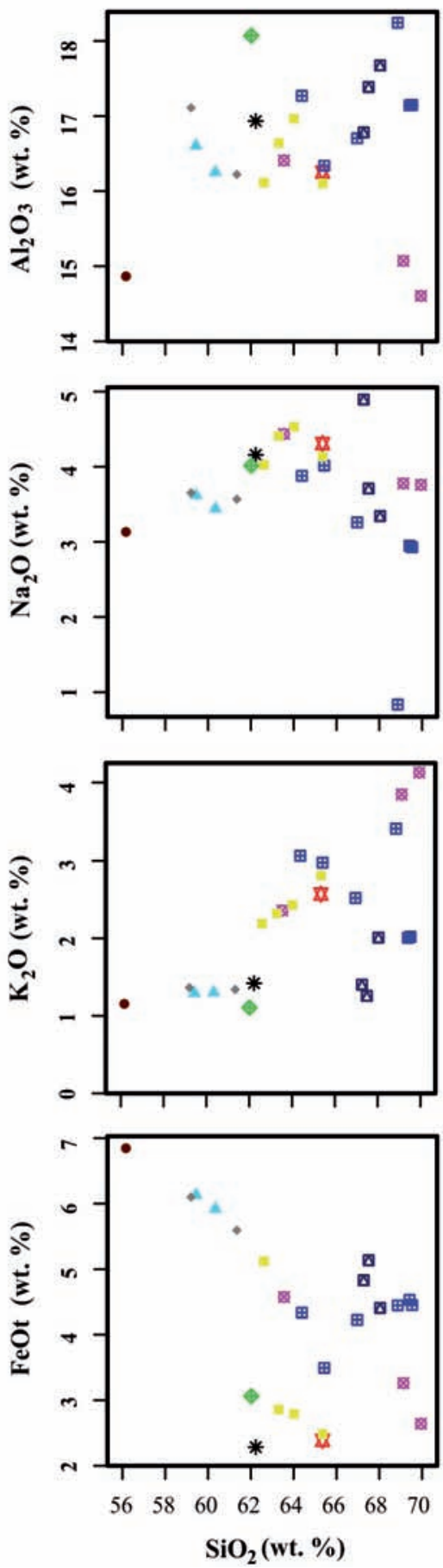

FIGURA 7. Diagramas de variación de elementos mayores vs contenido de sílice para los volcanes del Campo Volcánico Monogenético Villamaría-Termales (CVMVT). 
Según los diagramas tipo Harker para elementos traza, se puede observar una tendencia general positiva de $\mathrm{Ba}, \mathrm{Rb}, \mathrm{Zr}$, La y Ce, y negativa de $\mathrm{Sr}$ y $\mathrm{Cr}$ con respecto al aumento de $\mathrm{SiO}_{2}$ (aunque para el Cr existen pocos datos). Elementos como Y y Ni no muestran tendencias claras (FIGURA 8). Se destaca el bajo contenido de $\mathrm{Ba}$ en el Domo La Oliva, el alto contenido de Rb en
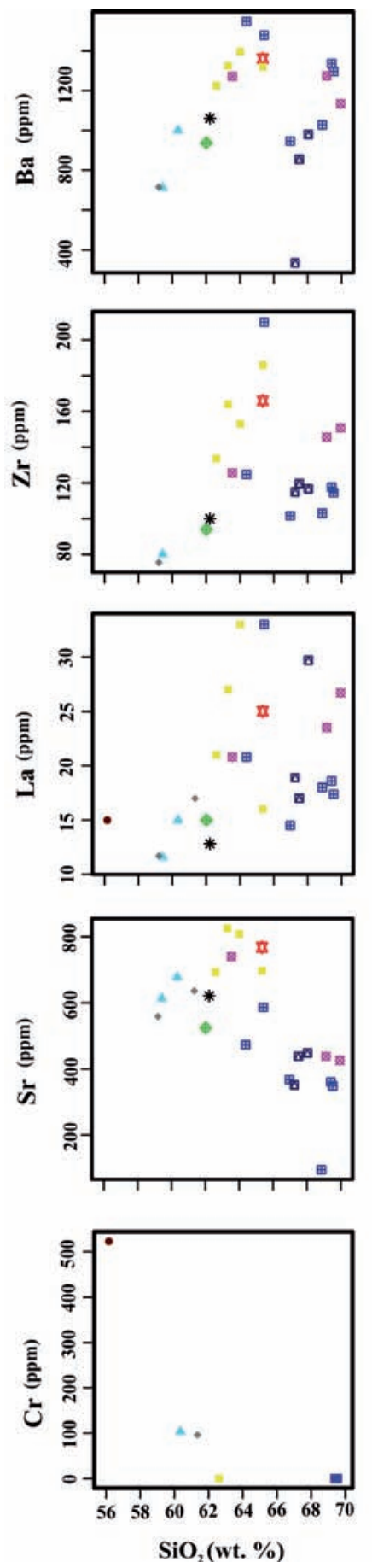

el Domo Amazonas, el alto contenido de Zr e Y y el bajo de Sr en el Domo Gallinazo, y el alto contenido de Ni en los domos Sancancio y La Esperanza en comparación con los otros volcanes del campo. Los domos La Laguna y Santana presentan bajo contenido de Ba, Rb, Zr, La y Ce (FIGURA 8).
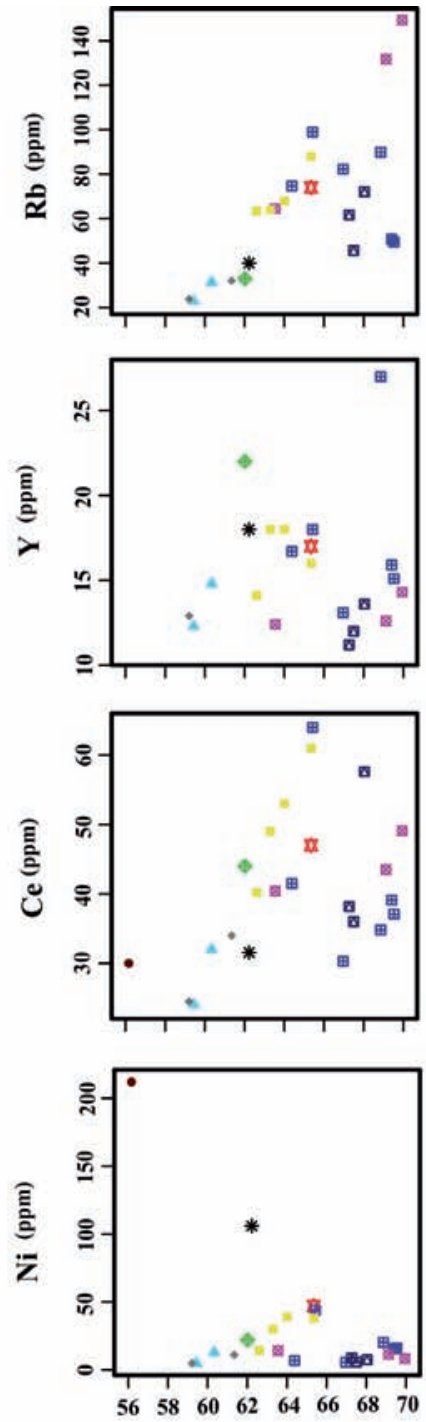

$\mathrm{SiO}_{2}$ (wt. \%)

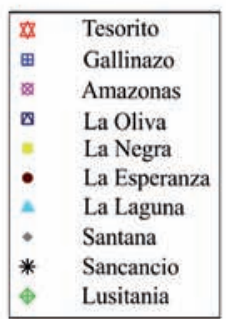

FIGURA 8. Diagramas de variación de elementos traza vs contenido de sílice para los volcanes del Campo Volcánico Monogenético Villamaría-Termales (CVMVT). 
En el diagrama de multielementos normalizado a corteza promedio de Weaver y Tarney (1984), se observa una tendencia composicional similar a los valores definidos para la composición de la corteza; no obstante, se observa también un enriquecimiento de elementos como $\mathrm{Ba}, \mathrm{Th}, \mathrm{U}, \mathrm{Tb}$ e $\mathrm{Y}$ en algunos de los volcanes, así como un empobrecimiento en $\mathrm{Nb}$ y $\mathrm{Zr}$ en otros. Una marcada anomalía negativa de Sr en una de las muestras del Domo Gallinazo es notable, al igual que una marcada anomalía positiva de $\mathrm{U}$ y $\mathrm{Nb}$ para algunas muestras de los domos Amazonas y La Esperanza, respectivamente (FIGURA 9).

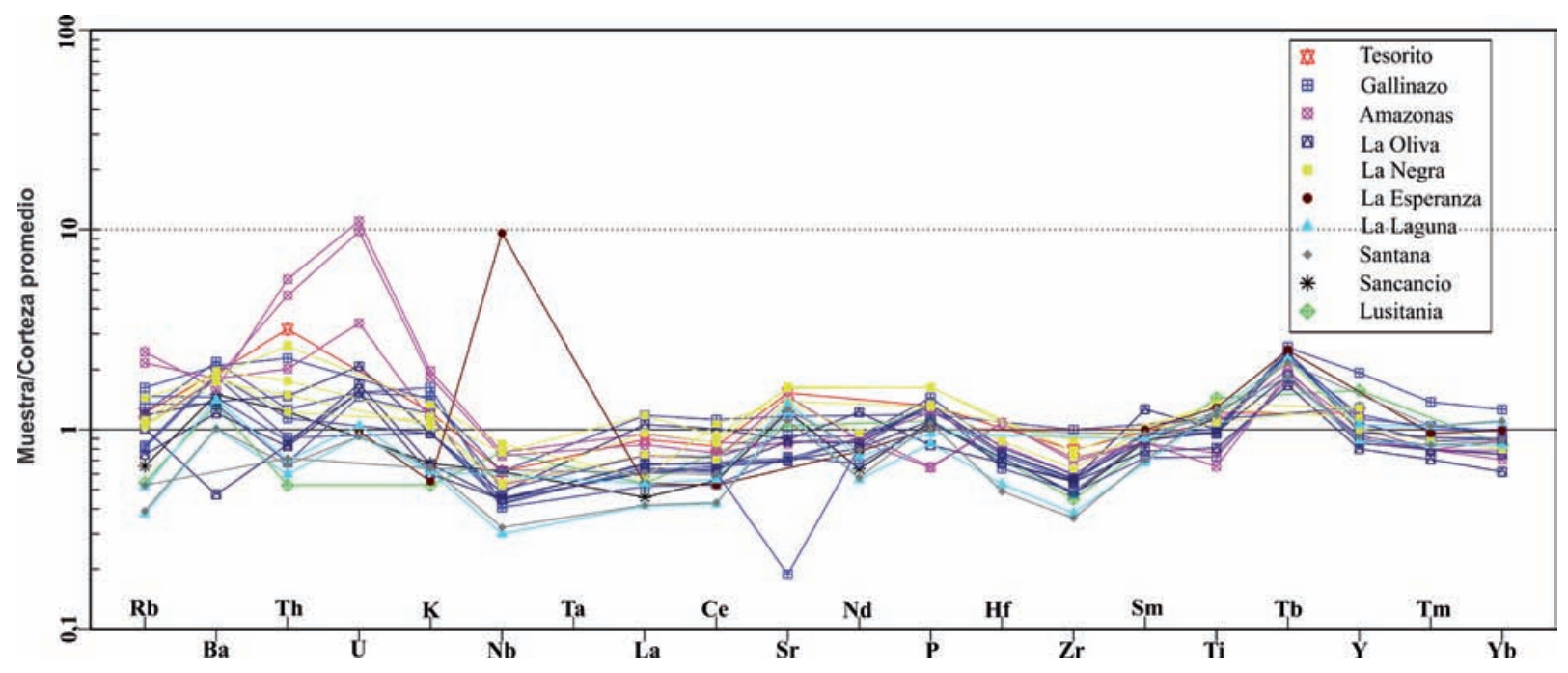

FIGURA 9. Diagrama de multielementos normalizado a corteza promedio de Weaver y Tarney (1984).

\section{DISCUSIÓN}

\section{Características composicionales}

El CVMVT muestra un espectro amplio y congruente en términos composicionales, con respecto al comportamiento de las expresiones volcánicas asociadas a la zona de subducción que contextualiza la región, además de la firma calcoalcalina característica. Las composiciones de los centros volcánicos del CVMVT varían entre andesitas y dacitas predominantemente, con la presencia ubicua de piroxeno, hornblenda y plagioclasa.

En general, los volcanes presentan una clara correlación entre la mineralogía y su comportamiento geoquímico. Por ejemplo, los centros eruptivos que presentan cristales de cuarzo (Grupo 2) tienen valores altos de $\mathrm{SiO}_{2}$ (67 - 69 wt.\%; FIGURA 6). Sin embargo, otros volcanes muestran un comportamiento geoquímico poco congruente con lo observado en los análisis petrográficos. Por ejemplo, los domos Amazonas y La Negra que se encuentran dentro de los centros eruptivos que presentan cristales de olivino (Grupo 1) presentan un alto contenido de $\mathrm{SiO}_{2}$ y álcalis (i.e. $63-$ 69 wt. \% y $\sim 7$ y 5 wt. \%, respectivamente; FIGURA 6), indicativo de un alto grado de evolución magmática. En este caso, la presencia de olivino puede interpretarse como incorporado durante el ascenso del magma, ya sea como antecristal o xenocristal (cf. Jerram y Martín, 2008). Esta misma interpretación (i.e. como xenocristal) se considera como la explicación para los cristales de feldespato potásico (Domo La Laguna) y cuarzo con bordes de reacción (Domo El Plato).

\section{Relación composicional de los centros volcánicos del CVMVT y el CVNR}

En un campo volcánico, la composición puede variar entre los diferentes centros eruptivos (e.g. Bacon et al., 1981; Schonwalder, 2015), así como también permanecer con características composicionales muy similares (e.g. Murcia et al., 2016). En campos volcánicos asociados a ambientes de subducción, el rango de composición tiende a ser relativamente más amplio (e.g. conos de escoria satélites en el volcán Llaima, Chile; Schonwalder, 2015), en comparación con ambientes de intraplaca (e.g. Auckland Volcanic Field; Smith y Allen, 1994) y/o de dorsal oceánica. Tal condición posiblemente se debe a que en estos últimos, la ausencia de cámaras magmáticas y el ascenso rápido del magma, restringen los procesos de cristalización fraccionada, contaminación cortical y/o mezcla de magmas, haciendo que los productos 
reflejen características composicionales similares a las del fundido primario (e.g. Murcia et al., 2016). En los campos volcánicos monogenéticos asociados a ambientes de subducción, los magmas usualmente evolucionados sugieren estancamiento en cámaras magmáticas que podrían estar asociadas con la generación de volcanes poligenéticos (e.g. Borrero et al., 2017). Así, los productos de ambos tipos de vulcanismo pueden compartir características geoquímicas y petrográficas similares (e.g. Schonwalder, 2015).

Composicionalmente, el CVMVT muestra una composición similar a la presentada por los productos
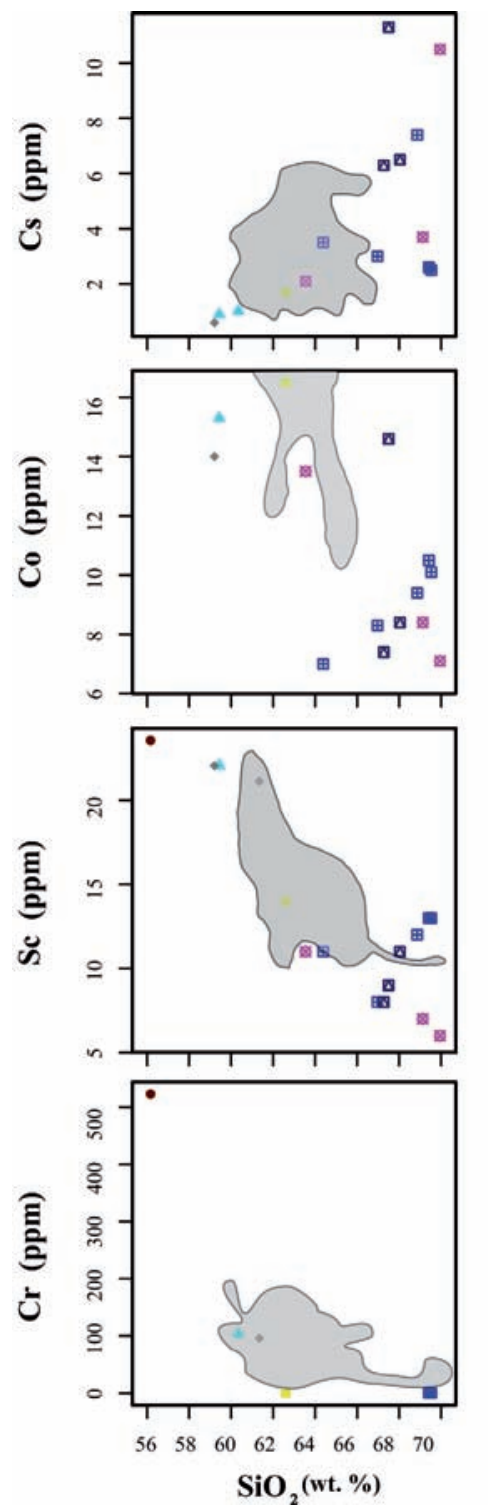

del CVNR a lo largo de su historia eruptiva (Thouret et al., 1990; Schaefer, 1995; Toro et al., 2008; Borrero et al., 2009). Por lo tanto, al comparar el comportamiento composicional del CVMVT con la composición de los productos asociados al CVNR, es posible identificar similitudes tanto en los óxidos mayores como en los elementos traza. De manera general, se observa que tanto para el CVMVT como para el CVNR, los productos se ubican en los campos de andesitas basálticas, andesitas y dacitas, con características de firma calcoalcalina de la serie media y alta en potasio (FIGURA 6A, 6B y 6C). El comportamiento de los elementos traza incluyendo los REE, son también similares (FIGURA 10).

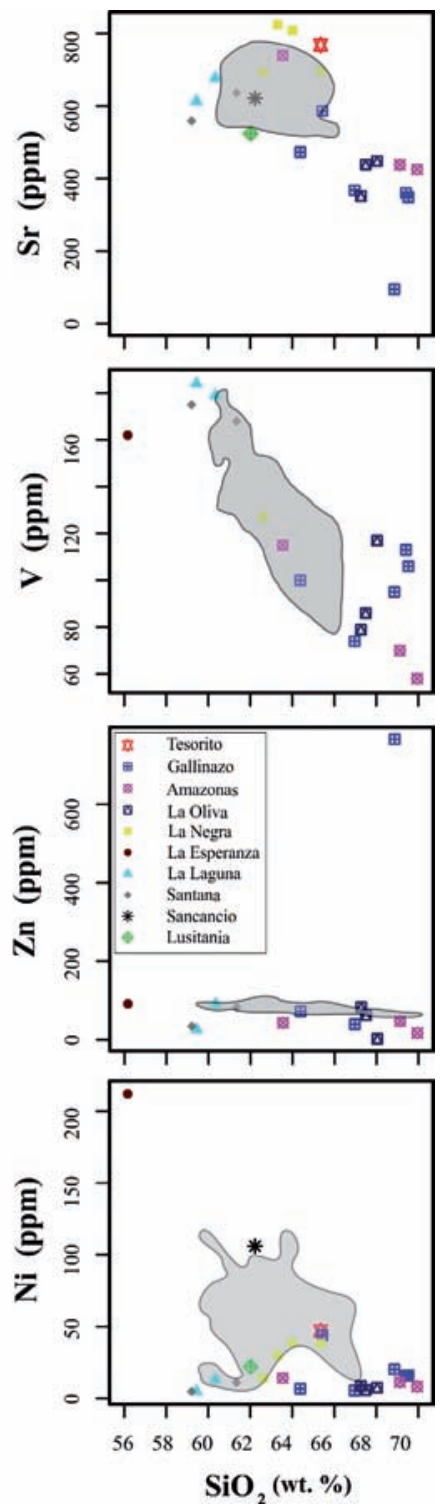

FIGURA 10. Diagramas binarios de $\mathrm{SiO}_{2}$ vs elementos traza. El sombreado gris corresponde a las tendencias para productos del CampoVolcánico Nevado del Ruiz (CVNR). 
En síntesis, las firmas composicionales del CVMVT y del CVNR son similares. Dicho resultado permite sugerir que el CVNR y el CVMVT son alimentados por un reservorio magmático común. Al respecto, Londoño (2016) propone un reservorio ubicado a $\sim 20-30 \mathrm{~km}$ de profundidad que alimenta el vulcanismo de toda la PVTSC (ver también Murcia et al., en prensa). En este trabajo se sugiere que este reservorio alimenta una cámara magmática más superficial (la cámara asociada al CVNR), y que a su vez alimenta también al CVMVT. Esta evolución está dominada por procesos comunes de ambientes de subducción descritos para diferentes volcanes de los Andes, tales como cristalización fraccionada, contaminación cortical y/o mezcla de magmas (e.g. Toro et al., 2008; Borrero et al., 2009; Martínez et al., 2014).

\section{Firma adaquítica en los volcanes del CVMVT}

Adaquita es un término para definir rocas ígneas asociadas con subducción de litósfera oceánica joven $(\leq 25 \mathrm{Ma})$ y aún caliente en $\operatorname{arcos}$ Cenozoicos (Kay, 1978; Defant y Drummond, 1990). Estas rocas son el producto de la fusión parcial de la placa oceánica subducente. Según Defant y Drummond (1990), las adaquitas se caracterizan por presentar valores de $\mathrm{SiO}_{2}$ $>56$ wt. $\%, \mathrm{Al}_{2} \mathrm{O}_{3}>15$ wt.\%, usualmente $\mathrm{MgO}<3$ wt.\%, bajo contenido de $\mathrm{Y}(<18 \mathrm{ppm})$, bajos contenidos de HREE (e.g. $\mathrm{Yb}<1,9 \mathrm{ppm})$, alto contenido de $\mathrm{Sr}(>400$ ppm), bajos contenidos de HFSE y valores de $\mathrm{Sr}^{86} / \mathrm{Sr}^{87}$ $<0,7040$. Al respecto, Castillo (2006) menciona que las adaquitas son prácticamente definidas como tal a partir de las relaciones $\mathrm{Sr} / \mathrm{Y}$ vs $\mathrm{Y}$ y La/ $\mathrm{Yb}$ vs $\mathrm{Yb}$.
El origen de las adaquitas puede ser explicado no solo por la fusión de la corteza oceánica como fue inicialmente propuesto, sino también por: 1) fusión parcial de la cuña astenosférica metasomatizada por fundidos provenientes de la placa subducente, 2) por la fusión parcial de la litósfera inferior, o 3) por la cristalización fraccionada y asimilación de la corteza continental inferior (e.g. Castillo, 2006).

En el CVMVT, algunos elementos químicos propuestos por Defant y Drummond (1990) $\left(\mathrm{SiO}_{2}, \mathrm{Al}_{2} \mathrm{O}_{3}, \mathrm{MgO}, \mathrm{Y}\right.$, $\mathrm{Yb}$ y $\mathrm{Sr}$ ), indican que algunos de los volcanes presentan firma adaquítica (Toro et al., 2008) (FIGURA 11). Aquellas muestras que se encuentran alejadas del campo corresponden a los centros volcánicos: Flujo de lava de Lusitania y Domo Gallinazo. Los elementos referencia existentes para el CVMVT son: $\mathrm{SiO}_{2}>56$ wt. $\%, \mathrm{Al}_{2} \mathrm{O}_{3}>15$ wt. $\%, \mathrm{MgO}<3$ wt. $\%$, Y entre 10 y 19 ppm, Yb entre 1,09 y 1,96 ppm, y Sr entre 518 y $848 \mathrm{ppm}$.

En cuanto al origen, aunque este alcance se sale de los objetivos del presente trabajo, es importante mencionar que para centros volcánicos como el CVNR, volcán Cerro Machín y cuerpos plutónicos de la región como el Stock de Manizales, el Stock del Hatillo y el Batolito de El Bosque también se ha presentado una firma adaquítica (Laeger et al., 2013, Martínez et al., 2014; Bustamante et al., 2017). En estos casos las relaciones $\mathrm{Sr} / \mathrm{Y}$ vs $\mathrm{Y}, \mathrm{La} / \mathrm{Yb}$ vs $\mathrm{Yb}$ y $\mathrm{Sr} / \mathrm{Y}$ vs $\mathrm{La} / \mathrm{Yb}$ muestran claramente la firma existente.
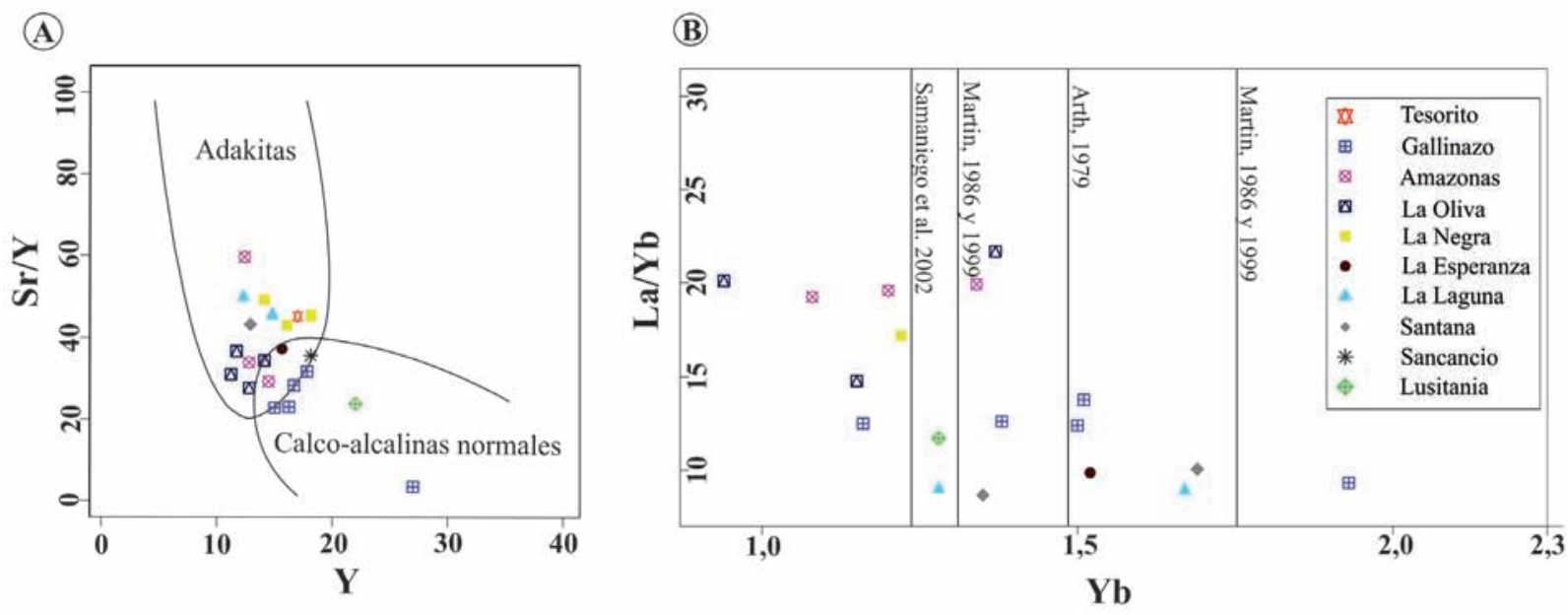

FIGURA 11. A. Diagrama $\mathrm{Sr} / \mathrm{Y}$ vs Y (tomado y modificado de Defant y Drummond, 1990) para discriminar entre adaquitas y rocas calco-alcalinas típicas. Las dos muestras más alejadas del campo adaquítico pertenecen a muestras de los centros volcánicos: Domo Gallinazo y Flujo de lava de Lusitania. Para discusión referirse al texto. B. Diagrama La/Yb vs Yb (tomado y modificado de Samaniego et al., 2005; en Martínez et al., 2014) para discriminar entre adaquitas y rocas calco-alcalinas. El límite entre los campos de adaquitas y rocas calco-alcalinas es diferente para varios autores: Arth (1979), Martin (1986, 1999), y Samaniego et al. (2005). No obstante, el valor de referencia usado aquí es el de Martín (1986, 1999) siguiendo a Castillo (2006). 
Para el caso del volcán Cerro Machín, el origen ha sido relacionado con la influencia del granate en la cristalización fraccionada asociada con la evolución de los magmas con contaminación cortical en la corteza inferior-media (Laeger et al., 2013). Para el caso de los cuerpos ígneos plutónicos el origen ha sido relacionado con la diferenciación del magma en el campo de estabilidad del granate (1,0-1,2 GPa) en la base de una corteza engrosada (Bustamante et al., 2017). Para el CVNR, Martínez et al. (2014) proponen un escenario que involucra la generación y evolución de los magmas a partir de la combinación de varios mecanismos, tales como: la fusión parcial de la cuña astenosférica, la cristalización fraccionada, la contaminación cortical, y la mezcla de magmas con un posible aporte de magma astenosférico. Es importante resaltar que la relación Sr/Y vs Y mostrada por Martínez et al. (2014), tiene una tendencia hacia el campo calcoalcalino; no obstante, la contaminación de las muestras con Y, como es aclarado por los autores, invalida esta relación. De esta manera, se sugiere que un posible origen de la firma adaquítica para el CVMVT, podría ser la asimilación de los cuerpos adaquíticos presentes en el basamento.

\section{CONCLUSIONES}

- Con base en las asociaciones mineralógicas, los centros volcánicos del CVMVT pueden subdividirse en tres grupos: 1 ) rocas con contenido de: olivino + piroxeno + anfíbol + plagioclasa, 2) rocas con contenido de: piroxeno + anfíbol + plagioclasa, y 3) rocas con contenido de: piroxeno + anfíbol + plagioclasa + cuarzo. Con base en los análisis químicos las rocas se clasifican como andesitas basálticas, andesitas, dacitas y riolitas.

- El CVMVT es un campo que presenta una firma adaquítica. Esta firma está posiblemente relacionada a procesos de asimilación cortical en profundidades de la corteza media/superior.

- Entender los procesos de generación de vulcanismo monogenético asociados con la PVTSC, sirve para entender el comportamiento de una futura erupción de un nuevo volcán monogenético, no solo como parte del CVMVT sino también de los otros campos volcánicos que se conocen en la provincia. Esto ayudaría a contribuir con futuras evaluaciones de amenaza volcánica para la región.

\section{AGRADECIMIENTOS}

Este trabajo fue desarrollado en el Instituto de Investigaciones en Estratigrafía - IIES (Universidad de Caldas); agradecemos a todos los integrantes por su apoyo y uso de materiales e instalaciones. Así mismo, agradecemos a Martha Gabriela Gómez Vasconcelos y John J. Sánchez por sus valiosos comentarios y recomendaciones en la revisión de este trabajo.

\section{REFERENCIAS}

Aguirre, O.R., y López, J.A. (2003). Cartografía geológica y petrografía del Stock de Manizales y su relación con sus rocas encajantes. Tesis, Programa de Geología, Facultad de Ciencias Exactas y Naturales, Universidad de Caldas, Manizales, Colombia.

Ancochea, E., Naranjo, J.L., Fuster, J.M., y Borrero, C. (1991). Geoquímica de las lavas antiguas del Volcán del Ruiz (Colombia), Sector Noroccidental. Simposio sobre Magmatismo Andino y su Marco Tectónico. Manizales, Colombia. Memorias Tomo I: 65-79.

Arth, J.G. (1979). Some trace elements in trondhjemites-their implications to magma genesis and paleotectonic setting. In: F. Barker (ed.). Trondhjemites, dacites, and related rocks (pp. 123-132). Vol. 6. New York: Elsevier. doi: 10.1016/B978-0-444-41765-7.50008-3.

Bacon, C.R., Macdonald, R., Smith, R.L., and Baedecker, P.A. (1981). Pleistocene high-silica rhyolites of the Coso Volcanic Field, Inyo County, California. Journal of Geophysical research: Solid Earth, 86(B11), 10223-10241. doi: 10.1029/ JB086iB11p10223.

Blanco-Quintero, I.F., García-Casco, A., Toro, L.M., Moreno, M., Ruiz, E.C., Vinasco, C.J., Cardona, A., Lázaro, C., and Morata, D. (2014). Late Jurassic terrane collision in the northwestern margin of Gondwana (Cajamarca Complex, eastern flank of the Central Cordillera, Colombia). International Geology Review, 56(15), 18521872. doi: 10.18273/revbol.v40n1-2018003. 
Bohórquez, O.P., Monsalve, M.L., Velandia, F., Gil, F., y Mora, H. (2005). Marco tectónico de la cadena volcánica más septentrional de la Cordillera Central de Colombia. Boletín de Geología, 27(44), 55-79.

Borrero, C., Toro, L.M., Alvarán, M., and Castillo, H. (2009). Geochemistry and tectonic controls of the effusive activity related with the ancestral Nevado Del Ruiz volcano, Colombia. Geofísica Internacional, 48(1), 149-169.

Borrero, C., Murcia, H., Agustín-Flores, J., Arboleda, M.T., and Giraldo, A.M. (2017). Pyroclastic deposits of San Diego maar, central Colombia: an example of a silicic magma-related monogenetic eruption in a hard substrate. In: K. Németh, G. Carrasco-Núñez, J.J. Aranda-Gómez, and I.E. Smith (ed.). Monogenetic Volcanism (pp. 361-374). London: Geological Society, Special Publications, 446.

Botero-Gómez, L.A., Osorio, P., Murcia, H., Borrero, C., y Grajales, J.A. (2018). Campo Volcánico Monogenético Villamaría-Termales, Cordillera Central, Andes Colombianos (Parte I): Características morfológicas y relaciones temporales. Boletín de Geología, 40(3), 85-102. doi: 10.18273/revbol.v40n3-2018005.

Bustamante, C., Cardona, A., Archanjo, C.J., Bayona, G., Lara, M., and Valencia, V. (2017). Geochemistry and isotopic signatures of Paleogene plutonic and detrital rocks of the Northern Andes of Colombia: A record of post-collisional arc magmatism. Lithos, 277, 199-209. doi: 10.1016/j.lithos.2016.11.025.

Cañón-Tapia, E. (2016). Reappraisal of the significance of volcanic fields. Journal of volcanology and Geothermal Research, 310, 26-38. doi: 10.1016/j. jvolgeores.2015.11.010.

Castillo, P.R. (2006). An overview of adakite petrogenesis. Chinese Science Bulletin, 51(3), 257268. doi: 10.1007/s11434-006-0257-7.

Defant, M.J., and Drummond, M.S. (1990). Derivation of some modern arc magmas by melting of young subducted lithosphere. Nature, 347(6294), 662665. doi: 10.1038/347662a0.

Gómez-Cruz, A., Sánchez, M., y Pardo-Trujillo, A. (1995). Edad y origen del “complejo metasedimentario Aranzazu-Manizales” en los alrededores de Manizales (departamento de Caldas, Colombia). Geología Colombiana, 19, 83-93.

Gómez, J., Montes, N.E., Novia, A., y Diederix, H. (2015). Mapa Geológico de Colombia. Escala, 1, 1000000. Servicio Geológico Colombiano.

González, H., y Londoño, A.C. (2002). Catálogo de las unidades litoestratigráficas de Colombia. Granodiorita de Manizales (K2E1gdm) Cordillera Central - Departamento de Caldas, 5 - 8.

González, L., y Jaramillo, C.M. (2002). Estudio neotectónico multidisciplinario aplicado a la Falla Villa María - Termales. Tesis, Facultad de Ciencias Exactas y Naturales, Programa de Geología, Universidad de Caldas, Manizales.

González-Garcia, J., and Jessell, M. (2016). A 3D geological model for the Ruiz-Tolima Volcanic Massif (Colombia): Assessment of geological uncertainty using a stochastic approach based on Bézier curve design. Tectonophysics, 687, 139157. doi: 10.1016/j.tecto.2016.09.011.

Irvine, T.N., and Baragar, W.R.A. (1971). A guide to the chemical classification of the common volcanic rocks. Canadian Journal of Earth Sciences, 8(5), 523-548. doi: 10.1139/e71-055.

Irving, E. (1971). La evolución estructural de los Andes más septentrionales de Colombia. Boletín Geológico, 19(2), 1-90. INGEOMINAS.

Janoušek, V., Farrow, C.M., and Erban, V. (2006). Interpretation of whole-rock geochemical data in igneous geochemistry: introducing Geochemical Data Toolkit (GCDkit). Journal of Petrology, 47(6), 1255-1259. doi: 10.1093/petrology/egl013.

Jerram, D.A., and Martin, V.M. (2008). Understanding crystal populations and their significance through the magma plumbing system. In: C. Annen and G.F. Zellmer (ed.). Dynamics of Crustal Magma Transfer, Storage and Differentiation (pp. 133148). Vol. 304. London: The Geological Society of London, Special Publications.

Kay, R.W. (1978). Aleutian magnesian andesites: melts from subducted Pacific Ocean crust. Journal of Volcanology and Geothermal Research, 4(1-2), 117-132. doi: 10.1016/0377-0273(78)90032-X. 
Laeger, K., Halama, R., Hansteen, T., Savov, I.P., Murcia, H.F., Cortés, G.P., and Garbe-Schönberg, D. (2013). Crystallization conditions and petrogenesis of the lava dome from the $\sim 900$ years BP eruption of Cerro Machín Volcano, Colombia. Journal of South American Earth Sciences, 48, 193-208. doi: 10.1016/j.jsames.2013.09.009.

Le Bas, M.J., Le Maitre, R.W., Streckeisen, A., and Zanettin, B. (1986). A chemical classification of volcanic rocks based on the total alkali-silica diagram. Journal of Petrology, 27(3), 745-750. doi: 10.1093/petrology/27.3.745.

Londoño, J.M. (2016). Evidence of recent deep magmatic activity at Cerro Bravo-Cerro Machín volcanic complex, central Colombia. Implications for future volcanic activity at Nevado del Ruiz, Cerro Machín and other volcanoes. Journal of Volcanology and Geothermal Research, 324, 156168. doi: 10.1016/j.jvolgeores.2016.06.003.

Maloney, K.T., Clarke, G.L., Klepeis, K.A., and Quevedo, L. (2013). The Late Jurassic to present evolution of the Andean margin: Drivers and the geological record. Tectonics, 32(5), 1049-1065. doi: 10.1002/tect.20067.

Martin, H. (1986). Effect of steeper Archean geothermal gradient on geochemistry of subduction-zone magmas. Geology, 14(9), 753756. doi: 10.1130/0091-7613(1986)14<753:EOS AGG>2.0.CO;2.

Martin, H. (1999). Adakitic magmas: Modern analogues of Archaean granitoids. Lithos, 46(3), 411-429. doi: 10.1016/S0024-4937(98)00076-0.

Martínez, L., Valencia L., Ceballos, J., Narváez, B., Pulgarín, B., Correa, A., Navarro, S., Murcia, H., Zuluaga, I., Rueda, J., y Pardo, N. (2014). Geología y estratigrafía del Complejo Volcánico Nevado del Ruiz. Informe final, Bogotá - Manizales Popayán. Servicio Geológico Colombiano. 853p.

Maya, M., y González, H. (1995). Unidades litodémicas en la Cordillera Central de Colombia. Boletín Geológico, 35(2-3), 43-57. INGEOMINAS.

McCourt, W.J. (1984). The geology of the Central Cordillera in the Department of Valle del Cauca, Quindío and NW Tolima. British Geological Survey, INGEOMINAS, Cali.
McCourt, W.J., Aspden, J.A., and Brook, M. (1984). New geological and geochronological data from the Colombian Andes: continental growth by multiple accretion. Journal of the Geological Society, 141, 831-845.

Mosquera, D. (1978). Geología del cuadrángulo K8 Manizales. Informe preliminar 1763. INGEOMINAS.

Murcia, H. (2015). Monogenetic volcanism in the western Arabian Peninsula: Insights from Late Quaternary eruptions in northern Harrat Rahat, Kingdom of Saudi Arabia. Ph.D. Thesis, The University of Auckland, Auckland, New Zealand.

Murcia, H., Borrero, C., and Németh, K. (in press). Overview and plumbing system implications of monogenetic volcanism in the northernmost Andes' volcanic province. Journal of Volcanology and Geothermal Research. doi: 10.1016/j. jvolgeores.2018.06.013.

Murcia, H., Lindsay, J.M., Németh, K., Smith, I.E.M., Cronin, S.J., Moufti, M.R.H., El-Masry, N.N., and Niedermann, S. (2016). Geology and geochemistry of Late Quaternary volcanism in northern Harrat Rahat, Kingdom of Saudi Arabia: implications for eruption dynamics, regional stratigraphy and magma evolution. In: K. Németh, G. Carrasco-Núñez, J.J. ArandaGómez, and I.E.M. Smith (ed.). Monogenetic Volcanism (pp. 173-204). Vol. 446. London: The Geological Society of London, Special Publications.

Németh, K. (2010). Monogenetic volcanic fields: Origin, sedimentary record, and relationship with polygenetic volcanism. In: E. Cañón-Tapia, and A. Szakács (ed.). What is a volcano? (pp. 4366). Vol. 470. New York: Geological Society of America. doi: 10.1130/2010.2470(04).

Nivia, A., Marriner, G.F., Kerr, A.C., and Tarney, J. (2006). The Quebradagrande Complex: A Lower Cretaceous ensialic marginal basin in the Central Cordillera of the Colombian Andes. Journal of South American Earth Sciences, 21(4), 423-436. doi: 10.1016/j.jsames.2006.07.002.

Peccerillo, A., and Taylor, S.R. (1976). Geochemistry of eocene calc-alkaline volcanic rocks from the Kastamonu area, northern Turkey. Contributions 
to Mineralogy and Petrology, 58(1), 63-81. doi: 10.1007/BF00384745.

Rayo, L. (2012). Evolución geoquímica y térmica del volcán Nevado del Ruiz, Colombia. M.Sc. Tesis, Universidad Nacional de Colombia, Bogotá, Colombia.

Restrepo, J.J., and Toussaint, J.F. (1988). Terranes and continental accretion in the Colombian Andes. Episodes, 7(3), 189-193.

Samaniego, P., Martin, H., Monzier, M., Robin, C., Fornari, M., Eissen, J.P., and Cotten, J. (2005). Temporal evolution of magmatism in the Northern Volcanic Zone of the Andes: The geology and petrology of Cayambe Volcanic Complex (Ecuador). Journal of Petrology, 46(11), 22252252. doi: 10.1093/petrology/egi053.

Schaefer, S.J. (1995). Nevado del Ruiz volcano, Colombia: magmatic system and evolution. Ph.D. Thesis, Arizona State University.

Schonwalder, D. (2015). The emplacement of satellite scoria cones at Llaima Volcanic System, Chile; The interaction between magmatic overpressure and the local tectonics. Ph.D. Thesis, State University of New York, Buffalo, New York, USA.

Smith, I.E., and Allen, S. R. (1994). Eruption styles and volcanic hazard in the Auckland Volcanic Field, New Zealand. Geoscience Reports of Shizuoka University, 20, 5-14.

Thouret, J.C., Cantagrel, J.M., Salinas, R., and Murcia, A. (1990). Quaternary eruptive history of Nevado del Ruiz (Colombia). Journal of Volcanology and Geothermal Research, 41(1-4), 225-251. doi: 10.1016/0377-0273(90)90090-3.

Toro, L.M., Alvarán-Echeverri, M., y Borrero-Peña, C.A. (2008). Rocas con afinidad adakítica al sur-este de Manizales: rasgos petrogenéticos y geoquímicos. Boletín de Geología, 30(2), 49-60.

Toussaint, J.F., y Restrepo, J.J. (1987). Límites de placas y acortamientos recientes entre los paralelos son y son, Andes Colombianos. Andean Geology, (31), 95-100. doi: 10.5027/andgeoV14n2-a10.

Vesga, C.J., y Barrero, D. (1978). Edades K/Ar en rocas ígneas y metamórficas de la Cordillera Central de Colombia y su implicación geológica. II Congreso Colombiano de Geología. Bogotá, Colombia.

Villagómez, D., Spikings, R., Magna, T., Kammer, A., Winkler, W., and Beltrán, A. (2011). Geochronology, geochemistry and tectonic evolution of the Western and Central Cordilleras of Colombia. Lithos, 125(3-4), 875-896. doi: 10.1016/j.lithos.2011.05.003.

Villagómez, D., and Spikings, R. (2013). Thermochronology and tectonics of the Central and Western Cordilleras of Colombia: Early Cretaceous-Tertiary evolution of the Northern Andes. Lithos, 160-161, 228-249. doi: 10.1016/j. lithos.2012.12.008.

Weaver, B.L., and Tarney, J. (1984). Empirical approach to estimating the composition of the continental crust. Nature, 310, 575-577. doi: 10.1038/310575a0.
Pablo Osorio

ORCID: 0000-0001-7523-9137

Luis Alvaro Botero-Gómez

ORCID: 0000-0002-0783-4097

Hugo Murcia

ORCID: 0000-0002-3570-1988

Carlos Borrero

ORCID: 0000-0002-4907-2078

Jeny Alejandra Grajales

ORCID: 0000-0002-6966-9971

Trabajo recibido: febrero 14 de 2018

Trabajo aceptado: mayo 29 de 2018 